ARTICLE

https://doi.org/10.1038/s41467-019-08349-0

\title{
Dual RNA-seq identifies human mucosal immunity protein Mucin-13 as a hallmark of Plasmodium exoerythrocytic infection
}

\author{
Gregory M. LaMonte ${ }^{1}$, Pamela Orjuela-Sanchez ${ }^{1}$ Jaeson Calla ${ }^{1}$, Lawrence T. Wang ${ }^{1}$, Shangzhong Li, ${ }^{1,2,3}$, \\ Justine Swann', Annie N. Cowell ${ }^{4}$, Bing Yu Zou', Alyaa M. Abdel-Haleem Mohamed ${ }^{5}$, \\ Zaira Hellen Villa Galarce ${ }^{6}$, Marta Moreno ${ }^{6,7}$, Carlos Tong Rios ${ }^{6}$, Joseph M. Vinetz ${ }^{4,6,8}$, Nathan Lewis ${ }^{1,2,3} \&$ \\ Elizabeth A. Winzeler ${ }^{1}$
}

The exoerythrocytic stage of Plasmodium infection is a critical window for prophylactic intervention. Using genome-wide dual RNA sequencing of flow-sorted infected and uninfected hepatoma cells we show that the human mucosal immunity gene, mucin-13 (MUC13), is strongly upregulated during Plasmodium exoerythrocytic hepatic-stage infection. We confirm MUC13 transcript increases in hepatoma cell lines and primary hepatocytes. In immunofluorescence assays, host MUC13 protein expression distinguishes infected cells from adjacent uninfected cells and shows similar colocalization with parasite biomarkers such as UIS4 and HSP70. We further show that localization patterns are species independent, marking both $P$. berghei and $P$. vivax infected cells, and that MUC13 can be used to identify compounds that inhibit parasite replication in hepatocytes. This data provides insights into host-parasite interactions in Plasmodium infection, and demonstrates that a component of host mucosal immunity is reprogrammed during the progression of infection.

\footnotetext{
${ }^{1}$ Department of Pediatrics, University of California, San Diego, School of Medicine, La Jolla, CA 92093, USA. ${ }^{2}$ Novo Nordisk Foundation Center for Biosustainability at the University of California, San Diego, La Jolla, CA 92093, USA. ${ }^{3}$ Department of Bioengineering, University of California, San Diego, La Jolla, CA 92093, USA. ${ }^{4}$ Division of Infectious Diseases, Department of Medicine, University of California San Diego, La Jolla, CA 92093, USA.

${ }^{5}$ Computational Bioscience Research Centre (CBRC) and Biological and Environmental Sciences and Engineering (BESE) division, King Abdullah University of Science and Technology (KAUST), Thuwal, Saudi Arabia. ${ }^{6}$ Laboratorio ICEMR-Amazonia, Laboratorio de Investigación y Desarrollo, Facultad de Ciencias y Filosofia, Universidad Peruana Cayetano Heredia, Lima, Peru. ${ }^{7}$ Present address: London School of Hygiene and Tropical Medicine, Department of Immunology and Infection, London, UK. ${ }^{8}$ Present address: Yale School of Medicine, Section of Infectious Diseases, Department of Internal Medicine, New Haven, CT, USA. These authors contributed equally to this work: Gregory M. LaMonte, Pamela Orjuela-Sanchez. Correspondence and requests for materials should be addressed to E.A.W. (email: ewinzeler@ucsd.edu)
} 
M alaria remains a significant global health problem with 214 million annual cases and up to a half million deaths in $2015^{1}$. The disease, caused by protozoan parasites of the genus Plasmodium, is transmitted when a female Anopheline mosquito takes a blood meal and injects infectious Plasmodium sporozoites. These sporozoites (typically less than 100) migrate to the liver where they invade hepatocytes. This exoerythrocytic infection develops asymptomatically in the infected hepatocytes over a period of 2-10 days, depending on the species of malaria parasite. The merosome released from the infected hepatocyte eventually bursts ${ }^{2}$, releasing tens of thousands of merozoites that are programmed to infect erythrocytes. The repeated infection and lysis of erythrocytes results in symptomatic disease, and for this reason, the erythrocytic stage has been the historical focus of drug discovery. On the other hand, the exoerythrocytic stage attracts attention due to the substantially reduced parasite burden. Unsurprisingly then, most malaria vaccine candidates (such as RTS,S/AS01 ${ }^{3}$, also known as Mosquirix) target the exoerythrocytic stage for this reason. In addition, while malaria is typically prevented through the use of insecticide-treated bed nets and treated with chemotherapy such as artemisinin combination therapies, there is a recognized need for new molecules that may protect against malaria and which might be formulated as a component in a Single Exposure, Radical Cure, and Prophylaxis medicine that could be used in a malaria-elimination campaign ${ }^{4}$.

From the perspective of host-parasite interactions, there are likely numerous possible host targets for therapeutic intervention. During the initial stage, the infected hepatocyte undergoes significant alteration yet does not undergo apoptosis. The parasite's metabolic needs are also likely to be considerable, given that one sporozoite can yield over 30,000 merozoites within a single infected host cell. It thus seems very likely that the parasite is releasing effectors into the host cell to control host cell behavior. This notion that the malaria parasite is modifying host-gene expression is heavily supported by studies in the related apicomplexan parasite, Toxoplasma gondii. Gene-expression studies in $T$. gondii have been used effectively to characterize the host response to infection, due to its high multiplicity of infection ${ }^{5,6}$. As observed in these studies, the parasite must carefully regulate immune activation and host-cell effector mechanisms (reviewed in ref. ${ }^{7}$ ) to establish infection. It is now known that multiple proteins, including ROP18 kinase ${ }^{8,9}$ and GRA15 ${ }^{10}$, are secreted into the host cell, altering host cell signal transduction and inflammation ${ }^{11}$.

In contrast to Toxoplasma, relatively little is known about molecules produced by hepatocytes in response to Plasmodium sporozoite infection, in part because of the difficulty associated with studying the exoerythrocytic stage (reviewed in ref. ${ }^{12}$ ). Plasmodium sporozoites form a parasitophorous vacuole within infected hepatocytes. Parasite infection is known to rely on multiple host molecules, including EphA2 and CD81, which have been shown to be essential for hepatocyte invasion ${ }^{13,14}$. Parasitesecreted molecules include LISP and IBIS1, which are secreted into hepatocytes in the Plasmodium berghei model ${ }^{15,16}$. Another candidate effector molecule is the circumsporozoite protein (CSP), an abundant protein that is shed from the parasite sporozoite surface. It was also shown that expression of recombinant CSP in HeLa cells regulates TNF-alpha dependent host-immune signaling and NF-kB translocation to the nucleus, for example ${ }^{17}$.

As with Toxoplasma, global gene-expression analysis of infected cells should be an effective way to identify host genes that play a role in Plasmodium exoerythrocytic infection. However, the low parasite to hepatocyte ratio also creates a low signal to noise ratio. This problem can be overcome using dual-RNA sequencing of flow-sorted infected host cells ${ }^{18}$, which analyzes host and pathogen transcriptomes simultaneously. In addition, the tremendous depth of coverage offered by current transcriptomic sequencing approaches allows for a deep examination of the data. Here, we have taken advantage of this dual-RNA sequencing approach in order to gain insights into Plasmodium liver-stage development. We have identified and partially characterized one host-factor, Mucin13, which is highly upregulated during host-cell infection by both $P$. berghei and Plasmodium vivax. In contrast to previously identified parasite markers of liver-stage infection, such as HSP70 or UIS4 ${ }^{19}$. Mucins, as a component of the mucosal immune system, serve a critical role in host innate immune defenses as an initial barrier to infection. MUC13, as one of the transmembrane mucins, has been shown to play important roles in defense against several forms of cancer ${ }^{20}$ and bacterial infections, such as those caused by Helicobacter pylori ${ }^{21}$. Mucosal immunity has not been shown to have a significant role in malaria (reviewed in ref. ${ }^{22}$ ), in part because most research is focused on the asexual blood stage. However, in this study, we display a novel interaction of innate immunity against parasite infection. Therefore, MUC13 may serve as a universal biomarker for Plasmodium infection, upregulated regardless of the species of infecting malaria parasite.

\section{Results}

Plasmodium infection significantly alters gene expression. To investigate host-pathogen interactions in Plasmodium exoerythrocytic stages, we conducted a dual RNA sequencing study, a strategy that has proven useful in identifying novel interactions in other intracellular parasitic organisms, including T. gondii and Leishmania major ${ }^{23-25}$. We initially used $P$. berghei, a cause of murine malaria, to infect the hepatoma cell line, Huh7.5.1, which had proven to be readily sortable using flow cytometry ${ }^{26}$. The Huh7.5.1 hepatocytes were first infected in vitro with $P$. berghei sporozoites expressing green fluorescent protein (GFP) regulated by an elongation factor-1 $\alpha$ promoter ${ }^{27}$ that is active throughout the 48 -h liver-stage infection ${ }^{26}$. Samples were collected at time 0 (uninfected hepatocytes and sporozoites before infection), 24 and $48 \mathrm{~h}$ postinfection (hpi), then infected and uninfected cells were separated using fluorescence-activated cell sorting. Altogether, approximately 10,000 cells of each type were collected, and subjected to a pilot study in which we determined that obtaining 20 million reads at $24 \mathrm{hpi}$, or 10 million reads at $48 \mathrm{hpi}$, would be sufficient to obtain an average $50 \times$ coding genome coverage for both host and P. berghei (Supplementary Figure 1). Huh7.5.1 experiments with 10,000 infected cells were then conducted in triplicate on three different days. To ensure that our expression patterns were not dependent on the human cell line used, we collected additional experiments with HepG2-CD81 cells (48 hpi infected and uninfected) and HC04 cells $(2 \times 48$ hpi infected and uninfected), both additional human hepatocyte cell lines able to support $P$. berghei infection ${ }^{28}$. Total RNA was isolated from the different populations and dual RNA-sequencing performed for both $P$. berghei and the host human hepatocyte-derived cell lines at each time point. Over 978 million total reads were obtained and aligned to either the $P$. berghei reference genome or the human reference genome, $72.8 \%(80.8 \%$ if not including sporozoite only samples) of which map to either the human or $P$. berghei genomes (Supplementary Data 1).

Altogether we detected transcripts with at least one sample having $>10$ reads for 21,941 of the 58,051 human genes and 4770 of the 5245 parasite genes (Parasite Read Counts-Supplementary Data 2, Human Host Read Counts-Supplementary Data 3). Principle component analysis showed, strong separation of the different cell types, but that expression patterns were generally consistent across experimental replicates $\left(R^{2}=0.86-0.89\right)$. 
Although our ultimate goal was to identify host biomarkers of infection, we first analyzed the gene-expression patterns for $P$. berghei as a control to ensure that our time point accurately reflected the expected parasite biology. In the parasite RNA sequencing data, PBANKA_050120 (UIS4) and PBANKA_040320 (CSP) were the most highly expressed within sporozoites (Supplementary Data 2). In contrast, PBANKA_071190 (HSP70) and PBANKA_100300 (LISP2), were the most highly expressed at 48 hpi (Supplementary Data 2). All are well-validated hepatic-stage markers ${ }^{15,29}$.

As anticipated, parasite gene-expression changes followed known patterns, including downregulation of genes at $48 \mathrm{hpi}$ (relative to sporozoites only) with known sporozoite function, such as CSP $\left(\log _{2} \mathrm{FC}\right.$ of $-9.6, p$ value $\left.3.61 \times 10^{-109}\right)$, CELTOS $\left(\log _{2} \mathrm{FC}\right.$ of -12.3 , $p$ value $\left.3.70 \times 10^{-78}\right)$, and UIS4 $\left(\log _{2} \mathrm{FC}\right.$ of $-9.3, p$ value $9.32 \times 10^{-219}$ ), combined with upregulation at 48 hpi of merozoite genes such as MSP1 $\left(\log _{2} \mathrm{FC}\right.$ of $3.8, p$ value $\left.2.78 \times 10^{-25}\right), M S R P 2\left(\log _{2} \mathrm{FC}\right.$ of $6.6, p$ value $\left.8.22 \times 10^{-23}\right)$, and SERA1 $\left(\log _{2} \mathrm{FC}\right.$ of $5.0, p$ value $\left.2.84 \times 10^{-46}\right)$ (Supplementary Data 4$)^{30}$. We also observed excellent concordance between our $P$. berghei expression data and reported P. falciparum quantitative microarray gene-expression studies, with the $P$. falciparum homolog of 5 of the 10 most highly expressed $P$. berghei sporozoite transcripts (by read count at $0 \mathrm{hpi}$ ) also being among the 10 most highly expressed P. falciparum transcripts (CSP, ETRAMP10.3/UIS4, TRAP, CELTOS, and HSP70-hypergeometric mean probability of association by chance $<1.51 \times 10^{-12}$ ) (Supplementary Data 2) ${ }^{31}$. In addition, 13 of the 20 most highly expressed $P$. falciparum sporozoite transcripts from that study $^{31}$ possessed homologs that were also highly expressed (within the top 100 genes) in our $P$. berghei dataset at $48 \mathrm{hpi}$ ( $p$ value $<2.82 \times 10^{-18}$ ) (Supplementary Data 2 ), demonstrating that the sporozoite transcriptional profile is largely shared between rodent and human Plasmodium species regardless of the experimental platform.

Interestingly, we observed less correlation between our studies and previous transcriptomic studies of Plasmodium yoelii liverstage parasites obtained directly from disassociated, infected mouse livers and analyzed using ratiometric two color 65-mer microarrays ${ }^{30}$. Examining the transition from 24 to $48 \mathrm{hpi}$ (this study) and 24 to $50 \mathrm{hpi}$ (P. yoelii), and ranking genes with data and with syntenic orthologs (1383 genes) in both species by fold change gave a Spearman rank correlation coefficient of only 0.022 (Supplementary Data 2). There were some similarities including the induction of Liver-Specific Proteins 1 and 2 in both datasets, although our data show a $>300 \mathrm{X}$ average $\log _{2} \mathrm{FC}$ at $48 \mathrm{hpi}$ for PBANKA_1003000 (LISP2), vs. a $\log _{2}$ FC of $1.7 \times$ for PY04387 in theirs. Another similarity was the strong induction of an uncharacterized gene (PY01013 and PBANKA_0519500) ranked at number 1 and 4, respectively in the two datasets (Supplementary Data 2). Although some differences are clearly platformdependent (e.g., RNAseq vs. two-color spotted microarrays, which have a smaller linear range ${ }^{30}$ ) there may be differences in the biology. For example, many of the most highly induced genes in our 48-h dataset relative to 24 -h had roles in merozoite function (e.g., MSP1, PBANKA_0831000, ranked 7 in our $P$. berghei dataset, and 1246 (of 1383)) in the $P$. yoelii dataset (Supplementary Data 2). Likewise, MSP7-like (PBANKA_1349000), ranked 1 in P. berghei and 1006 in P. yoelii (Supplementary Data 2), suggesting a slightly later stage of development at time of sampling for our dataset and potentially tighter synchronization for our in vitro study.

On the other hand, we found better concordance with the Tarun proteomic analysis ${ }^{30}($ Pearson correlation $=0.68)$ (Supplementary Data 2). The most abundant transcripts in our 48-h $P$. berghei RNAseq dataset corresponded to the genes with the most abundant spectra in the $P$. yoelii dataset. For example, 14 of the 30 top-ranked syntenic genes were shared between our 48 -h RNAseq dataset (average at $48 \mathrm{hpi}$ for Huh7.1) and the 40-h Tarun liver-stage proteomic dataset $\left(p\right.$ value $2.1 \times 10^{-11}$, hypergeometric mean test), despite the data coming from different species and different methods (Supplementary Data 2). Notably MSP1 was also highly expressed in the Tarun late-stage liver proteomic dataset (in contrast to the transcription study), ranking 16 out of 689 proteins in terms of spectra count at $50 \mathrm{hpi}$ (Supplementary Data 2).

After having demonstrated the validity of the experimental design, we turned our focus to our primary goal, the identification of host-factors involved in parasite development. In order to broadly examine host-cell gene-expression patterns, we first identified differentially expressed genes using pairwise comparisons between infected and uninfected cells focusing primarily on the 48-h time point (which was sampled with multiple hepatocyte lines) (Huh7.5.1 cells alone-Supplementary Data 4, all three cell lines (Huh7.5.1, HC04, and HepG2) combined at $48 \mathrm{H}-$ Supplementary Data 5). The expression profiles were next subjected to hierarchical clustering (Fig. 1a, Supplementary Data 3). The data showed, as predicted by principle component analysis, that there were dramatic differences between the different cell lines. However, subclusters could be identified that revealed genes that were consistently upregulated across cell lines with the most dramatic changes occurring $48 \mathrm{hpi}$.

In order to first classify the gene-expression changes, we determined whether specific functional classes were overrepresented in up and downregulated genes. Analysis of the 21,941 detected genes at the 48 -h time point showed that 840 human genes were upregulated (average $>2 \times$ change between pairs, $p$ value $<0.01)$ and 618 were downregulated using similar criteria (Supplementary Data 6 and 7). The sets of upregulated genes were compared to compiled lists of genes with known functions, including gene ontology groups, using Metascape ${ }^{32}$. We found that genes involved in host ribosome function ( $p$ value $3.23 \times 10^{-14}$ ), and DNA replication $\left(p\right.$ value $\left.3.46 \times 10^{-10}\right)$ were downregulated (Supplementary Data 6), indicating that the host cell has decreased the production of ribosomes and DNA replication machinery as it is presumably no longer dividing. The most strongly downregulated gene, $E N H O$, plays a role in the positive regulation of Notch signaling (Energy Homeostasis related, $-4.34 \times$ average $\log _{2} \mathrm{FC}, p$ value $1.11 \times 10^{-08}$ ). The chemokine ligand, CXCL10, which is a hallmark protein in the JAK-STAT, and alpha and gamma interferon pathways, was also highly downregulated $\left(-2.88 \log _{2} \mathrm{FC}, p\right.$ value $\left.4.68 \times 10^{-05}\right)$, as was thymidine kinase, a gene involved in DNA synthesis. Further analysis of dysregulated transcriptional pathways using an independent pathway analysis algorithm (Ingenuity pathway analysis ${ }^{33}$ ) in order to confirm the previous analysis, also indicated downregulation of the eIF2 signaling (Supplementary Figure 2).

The 840 upregulated genes were more difficult to classify. Transcript increases were observed for genes that play a role in general transcriptional pathways (Generic Transcription Pathway $-p$ value $2.0 \times 10^{-6}$ ), as well as genes with a role in the regulation of energy homeostasis ( $p$ value $1.32 \times 10^{-4}$ ) with enrichment in adipocytokine signaling pathway and gluconeogenesis, among others (Supplementary Data 7). These changes are presumably a reflection of the metabolic changes the host cell needs to make to support rapid parasite growth and replication. We also observe strong upregulation of mTOR signaling, whose upregulation has been shown to be necessary for hepatic development ${ }^{34}$, using Ingenuity pathway analysis. The most highly upregulated gene, however, was the human mucosal innate immunity gene, Mucin 13 (MUC13, average 16.51 upregulation, $p=7.84 \times 10^{-51}$ ), a gene 
a

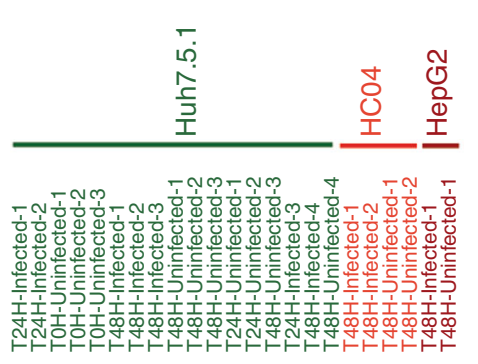

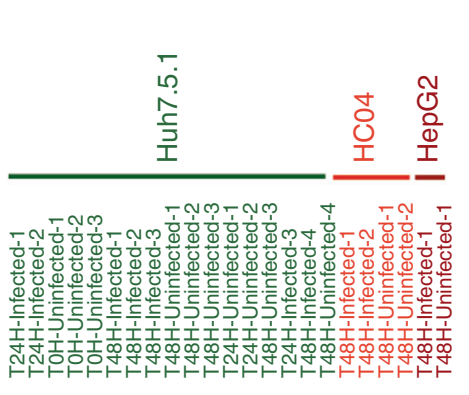
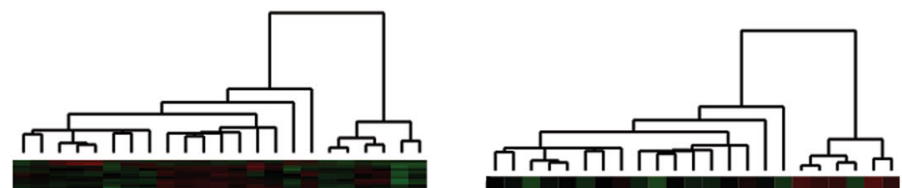

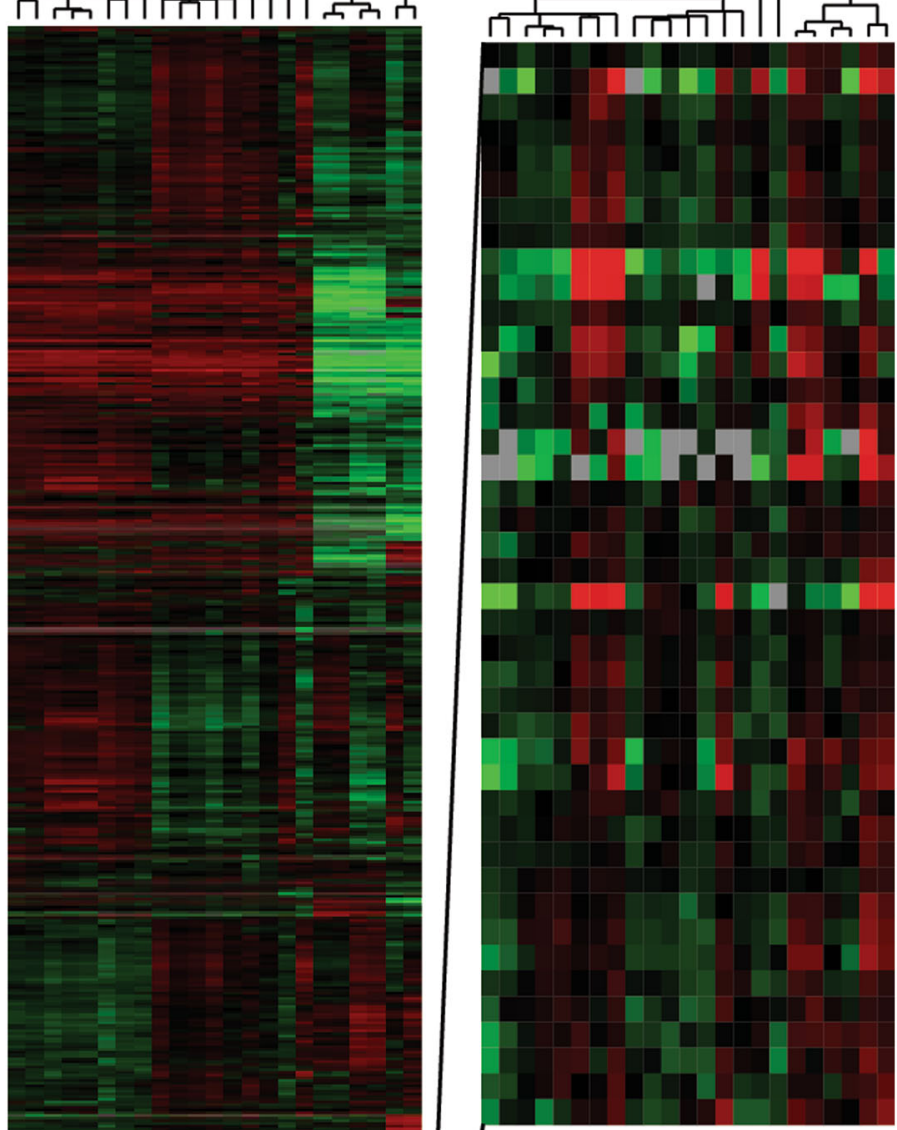

PHF7

FER1L4

CNNM2

MXI1

RIT1

LRRC37BP1

MYO1E

MUC13

NR4A3

CH507-42P11.8

AC005562.1

CCDC183-AS1

RP1-313|6.12

LTBP2

CATSPERG

SLC4A3

RP11-81A22.4

BCAN

C19orf66

ARSB

G6PC

FAM117A

MSMO 1

ACSS2

HSD17B7

RP11-390F4.3

CCDC183

MIR210HG

MAFF

RNF215

LDLR

GGT7

TPT1-AS1

MST

DICER1-ASI

SRCIN1

STX18-AS1

PRRT1

TMEM9B-AS1

USP27X-AS1

USP27X-AS1

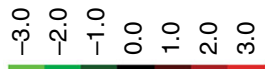

Infected vs uninfected

fold change $\left(\log _{2}\right)$

e

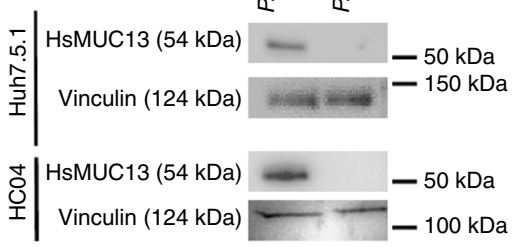


Fig. 1 Identification of differentially expressed host and parasite transcripts. a Hierarchical clustering of RNAseq samples based on gene-expression patterns in the indicated host hepatocytes and specified times postinfection. The color scale for the figure, as indicated by the scale bar in the lower left corner of Fig. 1a, indicates $\log _{2}$ fold changes from -3 to +3 . Data used to generate heatmap is located within Supplementary Data 3. $\mathbf{b} \vee V o l c a n o$ plot of gene-expression pattern vs. $p$ value, with the position of MUC13 indicated. c Confirmation of host factor upregulation via qPCR, with relative quantitation of the indicated host transcripts calculated using the $\Delta \Delta C T$ method, in the indicated hepatocyte cell types (data presented as mean \pm s.e.m, $n=3$ with individual biological replicates overlaid, ${ }^{*}=p$ value $<0.05,{ }^{\star \star \star}=p$ value $<0.001 . p$ values determined by two-tailed $t$ test). $\mathbf{d}$ Schematic of MUC13 protein structure and approximate antibody locations. Antibody \#1: AbCam \#Ab65109, Antibody \#2: LifeSpan BioSciences \#LS-C345092 (discontinued), and Antibody \#3: LifeSpan BioSciences \#LS-A8191. e Confirmation of MUC13 upregulation during infection via western blot

which is also upregulated at the mRNA level in response to $H$. pylori infection or IL- $1 \beta$ stimulation of MKN7 cells ${ }^{35}$ or treatment with the colitis-inducing agent, dextran sodium sulfate ${ }^{36}$.

MUC13 is upregulated during parasite hepatic infection. Because Mucin13 (MUC13) transcripts are found in some cancers including ovarian and colorectal cancers ${ }^{37}$ we next sought to confirm that this was not an artifact of using immortal hepatoma cells. We, therefore validated, using quantitative reverse transcription polymerase chain reaction (PCR), upregulation of the clearest example of an infection status marker gene (Fig. 1b), MUC13, in $P$. berghei-infected primary human hepatocytes, as well as in P. berghei-infected Huh7.5.1, HepG2, and HCO4 cells. In addition, we validated several additional genes (SLC22A8, RASSF9, and NEAT1) which were highly upregulated in Huh7.5.1 cells, yet not within this infection responsive cluster, as controls. In these four cell lines, we observed that only MUC13 $m R N A$ was consistently upregulated $(\sim 16 \times)$ (Fig. 1c).

MUC13 encodes a membrane bound mucin with abundant Oand N-glycosylation on its extracellular domain (Schematic: Fig. 1d) as well as EGF domains and has a molecular weight of 54 $\mathrm{kDa}$. To test whether we also observe upregulation of the MUC13 protein we performed western blot analysis using a commercially available polyclonal antibody that recognizes the C-terminal domain (Fig. 1d). Interestingly, MUC13 has been reported both as a $54 \mathrm{kDa}$ transmembrane protein and a putative, smaller 33 $\mathrm{kDa}$ soluble form in which the transmembrane domain has been cleaved $^{38}$. We clearly observed the $54 \mathrm{kDa}$ form (Fig. 1e), although we did not observe the lower molecular weight product (Supplementary Figure 3), indicating that the N-terminal extracellular domain may remain attached. In addition to recognizing the correct band, the MUC13 antibody showed protein upregulation in infected Huh7.5.1 and HCO4 cells (Fig. 1e), compared to uninfected co-sorted controls that were exposed to identical culture conditions.

MUC13 colocalizes with parasites during hepatic development. To assess whether MUC13 colocalizes with parasite in infected hepatocytes, we used two independent MUC13 antibodies (Antibody \#1: AbCam \#Ab65109, Antibody \#2: LifeSpan BioSciences \#LS-C345092 (discontinued)), including the antibody described above, and two independent parasite antibodies that react with heat-shock protein $70 \mathrm{kDa}$ (HSP70) and upregulatedin-sporozoites 4 (UIS4) (Fig. 2, Supplementary Figure 4). These two parasite antibodies stain the cytoplasm of developing exoerythrocytic form (EEF) schizonts ${ }^{39}$ and the parasite parasitophorous vacuolar membrane $e^{40}$, respectively. Our immunofluorescence (IFA) analysis showed that our MUC13 antibody recognized only parasite-infected cells. Interestingly, MUC13 is typically expressed on the outer cell membrane of host mucosal epithelial cells ${ }^{41}$ where it is found on apical membranes and forms part of the glycocalyx that presumably protects gut cells from pathogens. However, in parasite-infected cells the staining (Fig. 2, Supplementary Figure 4) largely colocalized with cytoplasmic parasite HSP70 (Supplementary Figure 4) and inside of UIS4 (Fig. 2), indicating that this host protein may be actively transported into the parasite cytoplasm. Although the cytoplasmic localization is surprising, it reflects the documented behavior of this protein in cancerous cells. It has been shown that MUC13 is cytoplasmic and overexpressed in metastatic colon cancer cells but apically located in normal adjacent control cells ${ }^{42}$. In addition, MUC13 is translocated from the apical membrane to the cytoplasm in colonic epithelium cells after treatment with the colitis-inducing agent, dextran sodium sulfate ${ }^{36}$. While this could be due to acquired cross-reactivity in stimulated cells, MUC13 antibodies reveal no MUC13 staining in knockout animals ${ }^{36}$. Although the apparent localization within the parasite cytoplasm could indicate cross-reactivity with parasite proteins, we found that MUC13 IFA levels closely correlate with transcript levels. We observed almost no MUC13 transcripts in our RNAseq at time zero and an IFA time course also showed no MUC13 staining at two hpi, during which time parasites were present and could be readily detected by HSP70 antibody staining (Fig. 3). The intensity of MUC13 staining was also directly proportional to MUC13 RNA levels, with a very modest induction at $24 \mathrm{hpi}$, and increasing to strong induction at $48 \mathrm{hpi}$ (Fig. 3). This induction of MUC13 also seems to be specific to exoerythrocytic infection, as IFA analysis for MUC13 in asexual blood stage parasites (with MUC13 Antibody \#2-LifeSpan Biosciences \#LS-C345092) shows no colocalization with parasites, only a faint MUC13 signal along the RBC membrane (Supplementary Figure 5). In addition, staining with a third, independently derived, commercial rabbit polyclonal antibody to the MUC13 extracellular domain (MUC13 Antibody \#3-LifeSpan BioSciences \#LS-A8191) showed nearly identical co-staining patterns (Supplementary Figure 6) to the other two antibodies.

A significant concern remained whether MUC13 was a marker for human malaria infection, or was simply limited to the rodent model. In order to validate the utility of MUC13 as a marker of a human malaria infection, we next examined the subcellular localization of MUC13 in cells infected with the human parasite, $P$. vivax. Symptomatic $P$. vivax malaria patients were recruited from the Loreto region of Peru using an approved human subjects' protocol and asked to provide a blood sample. This patient blood was washed and used to feed colonized female Anopheles darlingi mosquitos ${ }^{43}$ using a standard membranefeeding assay. After 14 days, salivary glands were dissected and the resultant sporozoites were harvested and incubated with HC04 cells for 7 days. IFA microscopy demonstrated that MUC13 also colocalizes very strongly with $P$. vivax HSP70 in HC04 cells (Supplementary Figure 4), indicating that MUC13 is a marker of human malaria infection as well. Similar to the rodent parasite, MUC13 signal covers the parasite, as would be observed if MUC13 is localized to the cytosol and nuclear membrane of developing merozoites of the developing exoerythrocytic parasite.

MUC13 is a quantifiable host marker of parasite infection. Species-specific parasite antibodies are typically used in liver- 
a

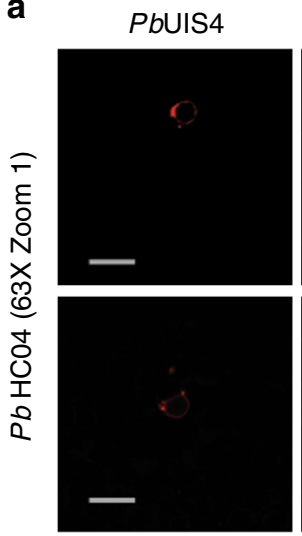

b

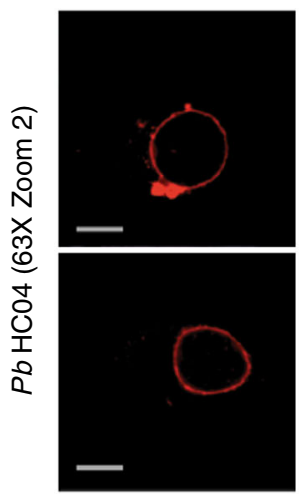

C

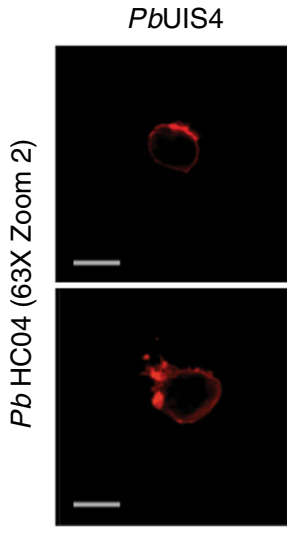

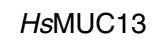
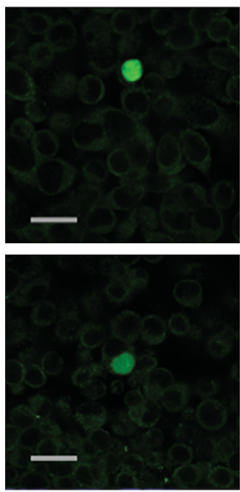

HsMUC13

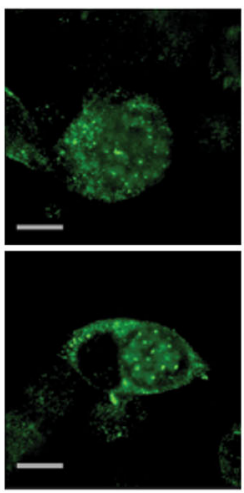

No HsMUC13 Ab control

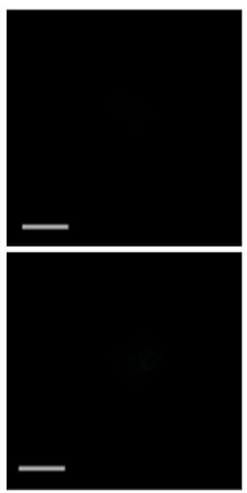

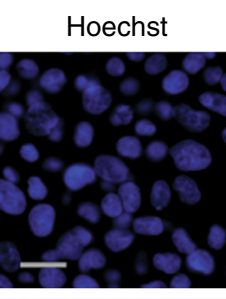

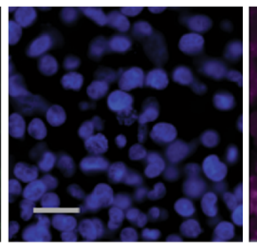

Hoechst

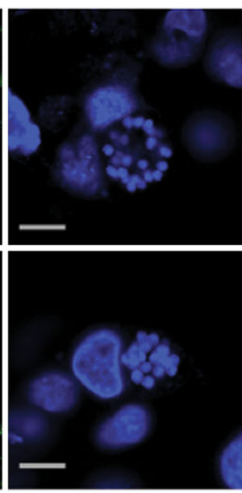

Hoechst

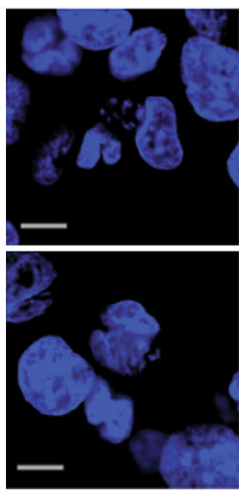

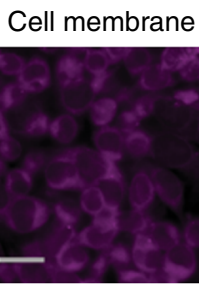

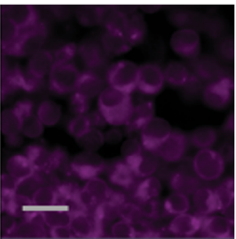

Cell membrane
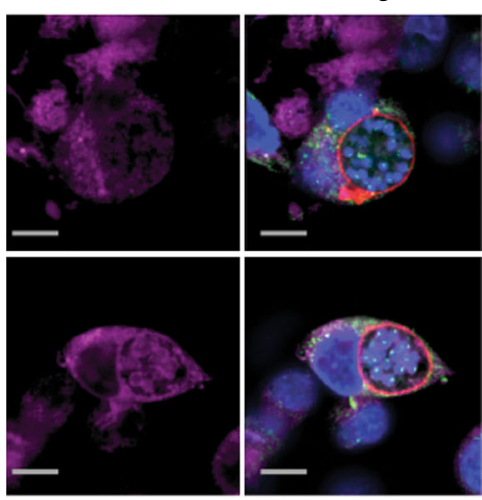

Cell membrane

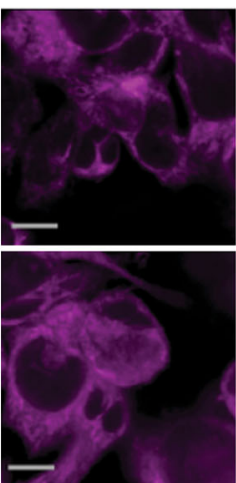

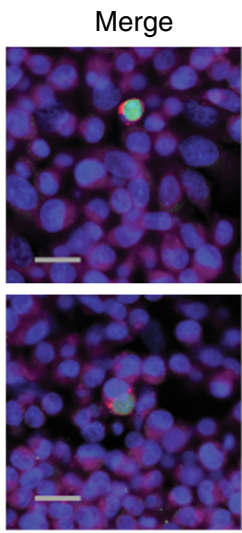

Merge

Fig. 2 Expression and localization of MUC13 in Plasmodium infected hepatoma cells. a, b Confocal microscopy images of representative HC04 liver cells infected with Plasmodium parasites $48 \mathrm{hpi}$ at two different magnifications. c Control Confocal microscopy images of representative HCO4 liver cells infected with Plasmodium parasites 48 hpi without MUC13 antibody being used. Cells were labeled using a rabbit polyclonal antibody (dilution 1:500, $1 \mathrm{mg} / \mathrm{ml}$ stock) against the intracellular region of MUC13 (MUC13 antibody \#1-AbCAM \#Ab65109) and visualized with a goat anti-rabbit Alexa Fluor 488 (green); CellMask deep red was used for plasma membranes (Cell Membrane-magenta). P. berghei EEFs were labeled using a goat polyclonal (dilution 1:200, $1 \mathrm{mg} / \mathrm{ml}$ stock) against PbUIS4 (LS-204260, LifeSpan Biosciences) and visualized with a bovine anti-goat secondary antibody (Alexa Fluor 647, red), respectively. Nuclei were labeled with Hoechst 33342 (blue). Merged images between HsMUC13, UIS4 and Hoechst are shown. Scale bars 50 (zoom 1) or 10 (zoom 2) $\mu \mathrm{m} ; 63 \times$ oil objective

stage imaging assays to measure parasite killing in response to drug candidates ${ }^{28}$. To assess whether MUC13 antibodies could be used as a substitute for parasite antibodies in imaging assays, we performed automated microscopy on $P$. berghei and $P$. vivax infected cultures after staining with parasite and the MUC13 antibody. HsMUC13 antibodies indicated nearly identical levels of parasitemia relative to parasite HSP70 (Fig. 4a). The value of MUC13 for parasite detection was also seen in dose response testing, as MUC13 was able to detect the decrease in parasite levels after dose-titration with two control compounds, atovaquone and puromycin (Fig. 4b), which yielded $\mathrm{EC}_{50}$ values equivalent to those determined using parasite HSP70 (Fig. 4c). Similar compound responses were also observed between HSP70 and MUC13 in parasites treated with compound at 12 and $24 \mathrm{hpi}$ (Supplementary Figure 7). The fluorescent signal from MUC13 antibody staining clearly demonstrates the effect of atovaquone 

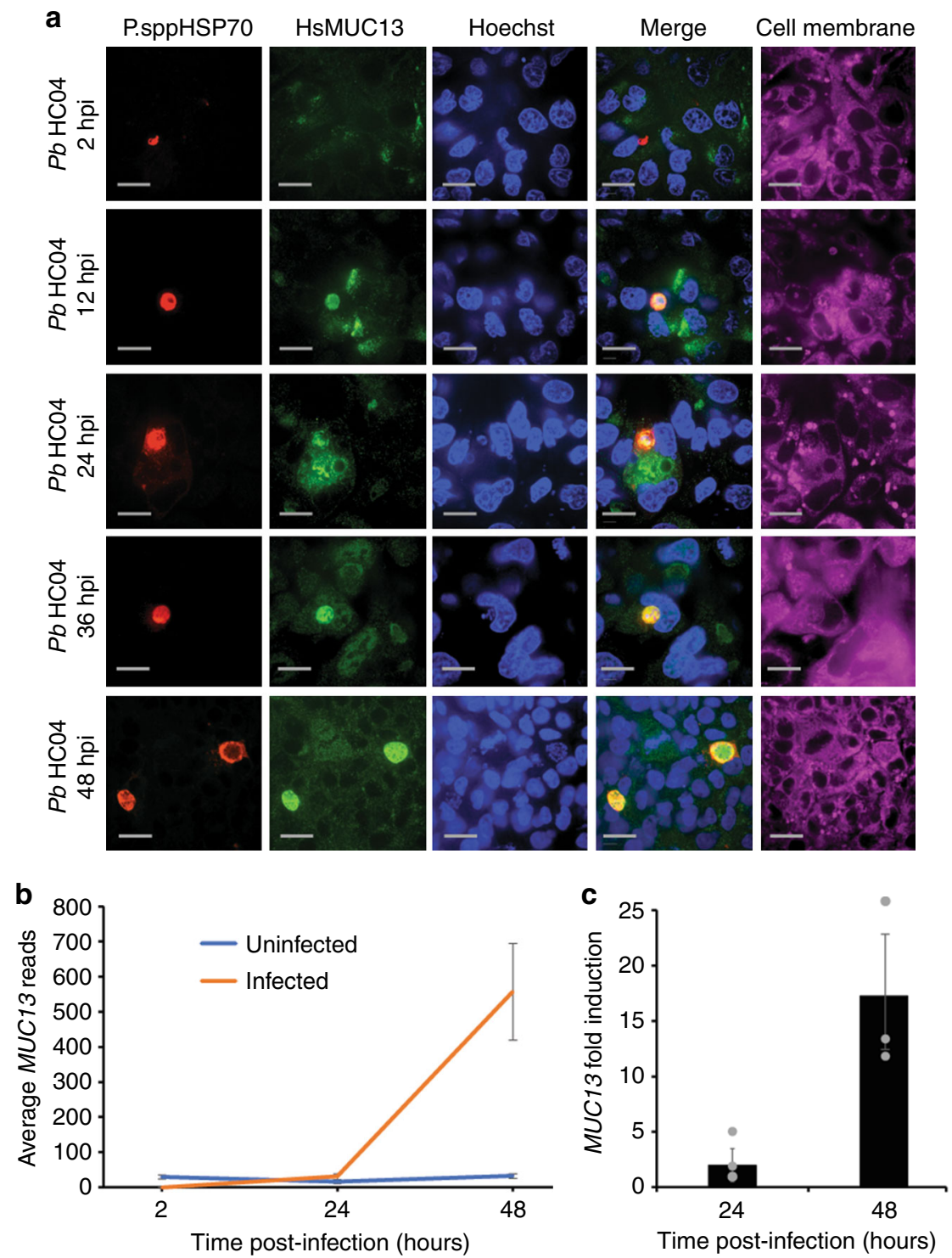

Fig. 3 Temporal detection of MUC13 in $\mathrm{HCO} 4$ cells infected with P. berghei. a $\mathrm{HCO} 4$ cells were infected with P. berghei sporozoites and then fixed and stained at 2, 12, 24, 36, and $48 \mathrm{hpi}$. Infected cell cultures were stained using a 1:500 dilution $(1 \mathrm{mg} / \mathrm{ml}$ stock) of mouse polyclonal antibody to P.spp HSP70 (see methods) and a 1:500 dilution of rabbit polyclonal antibody to MUC13 intracellular domain (MUC13 antibody \#2-LifeSpan BioSciences \#C345092). Primary antibody localization was visualized with goat anti-mouse (Alexa Fluor 647, red) and goat anti-rabbit (Alexa Fluor 488, green) secondary antibodies, respectively. Nuclei were stained with Hoechst 33342 (blue) and cell membranes with CellMask deep red (magenta). Scale bars $10 \mu m$; $60 \times$ oil objective. $\mathbf{b}$ The total reads, from the Huh7.5.1 RNA seq samples 1-3, for MUC13 at the 5 indicated time points $(0 \mathrm{~h}$ uninfected, $24 \mathrm{~h}$ infected, $24 \mathrm{~h}$ uninfected, $48 \mathrm{~h}$ infected, and $48 \mathrm{~h}$ uninfected). c The fold-induction of MUC13, based upon the total read count in panel B, at 24 and $48 \mathrm{hpi}$, presented as a ratio of Infected:Uninfected. Data presented as mean \pm s.e.m, $n=3$ with individual biological replicates overlaid

upon parasite growth, as $P$. berghei parasites treated with atovaquone very early in the $P$. berghei lifecycle $(2 \mathrm{hpi}$, when atovaquone is most effective ${ }^{26}$ ) demonstrate a marked decrease in size (Fig. 4d) and infection rate when compared with later or no drug treatment. This also indicates that the MUC13 induction begins prior to $48 \mathrm{hpi}$, since parasites treated with a lethal dose of atovaquone at $12 \mathrm{hpi}$ still exhibit MUC13 expression. These data show that MUC13 can substitute as a biomarker of parasite hepatic infection in drug discovery assays.

Although the MUC13 gene can be disrupted in mice it has been shown, nevertheless, to protect human epithelial cells against inflammation by inhibiting cellular apoptosis ${ }^{20}$. To determine whether MUC13 may play a role in Plasmodium survival and persistence, we used two parallel approaches to inhibit MUC13: a traditional stable pooled shRNA approach, delivered by lentivirus, to independently knockdown human MUC13 in both HC04 and Huh7.5.1 cells; and CRISPR/Cas9 to knockout MUC13 in clonal HC0 4 cell lines. The shRNA approach generated an observed $60 \%$ knockdown in MUC13 protein levels (Fig. 5a), while the knockout approach yielded multiple clones exhibiting no MUC13 protein expression (Fig. 5a). Stable MUC13 knockdown and knockout $\mathrm{HCO} 4$ cells were established, and when infected with $P$. berghei parasites expressing luciferase, we observed no decrease in either parasite load (via luciferase) or parasite number (via flow cytometry) at $48 \mathrm{hpi}$ (Fig. 5b). This lack of decrease in parasite number likely indicates that MUC13 is not required for parasite growth in vitro, suggesting that it may play a role in vivo, potentially during merosome release or to assist in immune invasion. Imaging of parasites infecting shMUC13 knockdown or knockout cells by IFA (Fig. 5c) indicated that the MUC13 


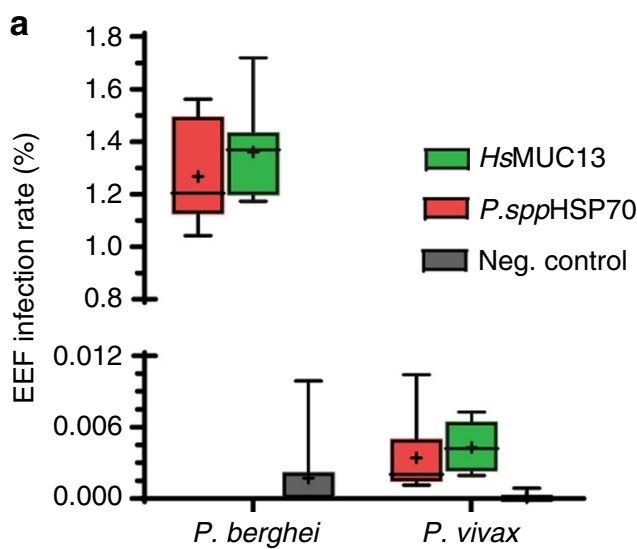

C

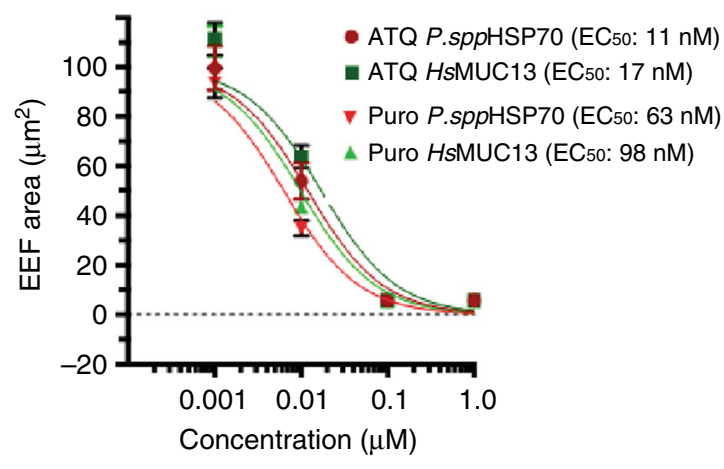

b

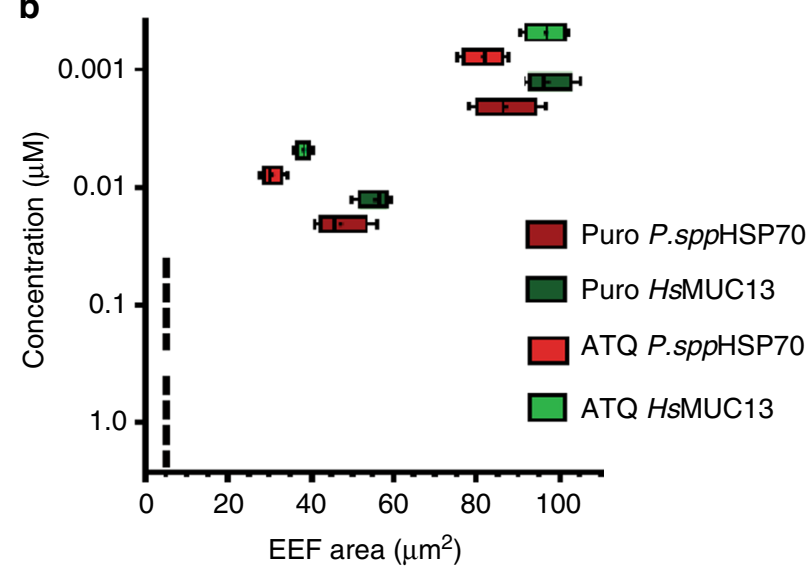

d

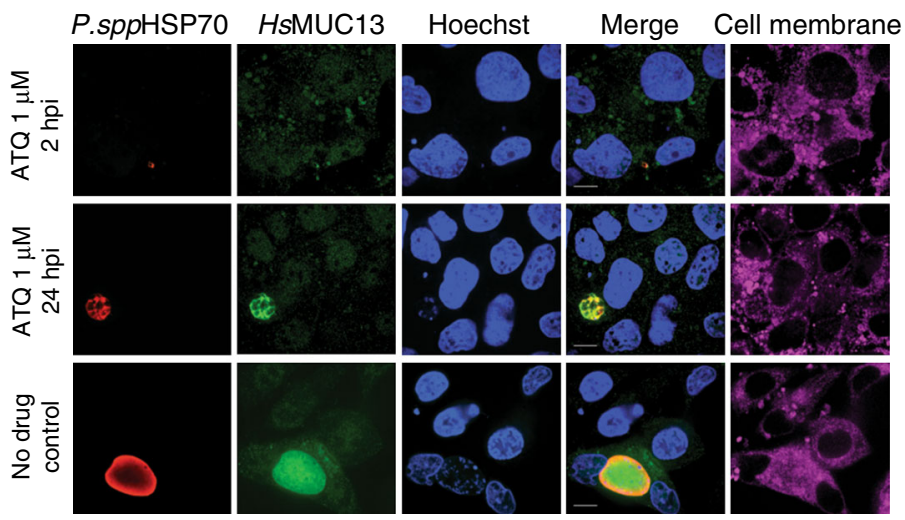

Fig. 4 MUC13 as a quantitative biomarker of Plasmodium EEF infection. a Counts of $P$. berghei or $P$. vivax EEF in HCO4 cells by indirect immunofluorescence. Negative controls with no primary antibodies were included. Parasite burden was estimated by counting at least 240,000 cells, via high content imaging. Data $(n=3)$ presented with the mean indicated by a " + " and error bars indicating the 5-95\% confidence interval. b Effect of atovaquone (ATQ) and puromycin (PURO) treatment ( $2 \mathrm{hpi}$ ) on cell area (growth) of $P$. berghei EEF in HCO4 at 48 hpi. Data $(n=4)$ presented with the mean indicated by a " + " and error bars indicating the $5-95 \%$ confidence interval. c Dose-response curves of $P$. berghei EEF in HCO4 cells for atovaquone (ATQ) and puromycin (PURO). 95\% confidence interval for $\mathrm{EC}_{50 \mathrm{~s}}=$ ATQ P. spp HSP70, 8.98-15.82; ATQ HsMUC13, 11.18-26.05; PURO HsMUC13, 6.29-15.29; PURO P. spp HSP70, 5.01-7.92. Data presented as mean $\pm \mathrm{SD}, n=2$ with 5-95\% confidence intervals indicated. d Representative images of $P$. berghei EEF in HCO4 cells (48 hpi) treated ( $2 \mathrm{hpi}$ ) with $1 \mu \mathrm{M}$ of atovaquone, puromycin, or DMSO. P. berghei was labeled with $P$. ssp HSP70 mouse polyclonal antibody (dilution 1:500, $1 \mathrm{mg} / \mathrm{ml}$ stock). $\mathrm{HCO} 4$ cells were labeled with a rabbit polyclonal antibody (dilution 1:500, $1 \mathrm{mg} / \mathrm{ml}$ stock) recognizing the intracellular region of $\mathrm{HsMUC}_{\mathrm{M}} \mathrm{B}$ (MUC13 antibody \#2-LifeSpan BioSciences \#LS-C345092). Primary antibody detection was performed with goat anti-mouse (Alexa Fluor 647, red) and goat anti-rabbit (Alexa Fluor 488, green) antibodies. Nuclei and cell membranes were stained with Hoechst 33342 (blue) and CellMask deep red (magenta), respectively. Scale bar $10 \mu \mathrm{m} ; 100 \times$ oil

antibody is specific for the protein, as staining in both the parasites and in the surrounding infected cell.

\section{Discussion}

As the goal of malaria eradication moves forward, the need for biomarkers to identify individuals infected with malaria without clinical symptoms will be essential. Our data illustrate the importance precise synchronization and deep sequencing, the lack of which may mask differences. For example, Albuquerque et al. $^{44}$ had previously performed a microarray-based study using infected mouse livers at $40 \mathrm{hpi}$ using $P$. yoelii, as well as $P$. berghei infection of the mouse cell line, Hepa1-6 at $24 \mathrm{hpi}$, and showed relatively few genes that were consistently changed between the species and time points.

Likewise, another very recent study also used several fluorescence-activated cell sorting (FACS)-sorted hepatocyte cell lines and GFP-expressing P. berghei and identified Aquaporin 3 as differentially expressed in HepG2 cells at $24 \mathrm{hpi}^{45}$. Our data also showed significant upregulation of Aquaporin 3 at $48 \mathrm{hpi}$ in HepG2 cells and HC04 cells (Supplementary Data 4). Likewise, their study observed upregulation of Mucin 13 during late-stage parasite development. Despite being one of the most highly upregulated genes in their study (ranked 4 when considering 18,720 genes that were analyzed in both studies) it was not 1 of the 5 genes considered statistically significant in the RNAseq analysis of 48 hpi Huh7.5.1 cells (adjusted $p$ value $=0.63$ in their study, vs. adjusted $p$ value $=1 \times 10^{-53}$ in ours). The fact that very few genes changed to a level that reached statistical significance in this study (vs. $>3000$ with an adjusted $p$ value of less than 0.05 and a fold change of greater than 2 in ours) could also be due to experimental variation across their replicates, loss of synchronization, or potentially sample processing differences.

Interestingly, we found some notable similarities with an earlier study which focused on timepoints at 30,60 , and 180 min postinfection in human cells ${ }^{46}$. This study compared bulk (not flowsorted) human HepG2-A16 cell responses after infection with irradiated and nonirradiated Plasmodium falciparum sporozoites as well as after exposure to salivary gland material. Systematic searches showed that a number of genes that they showed were changing between conditions were also changing between conditions in our study. Of the 742 differentially expressed 
a

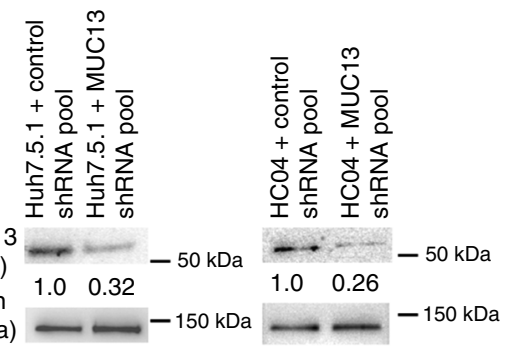

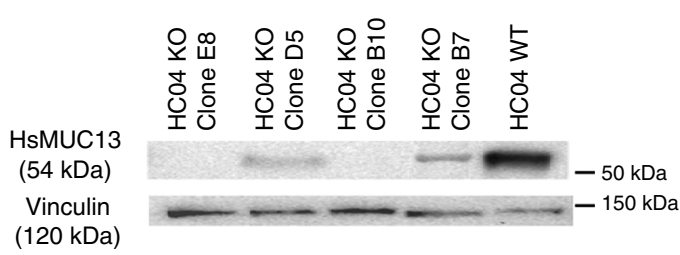

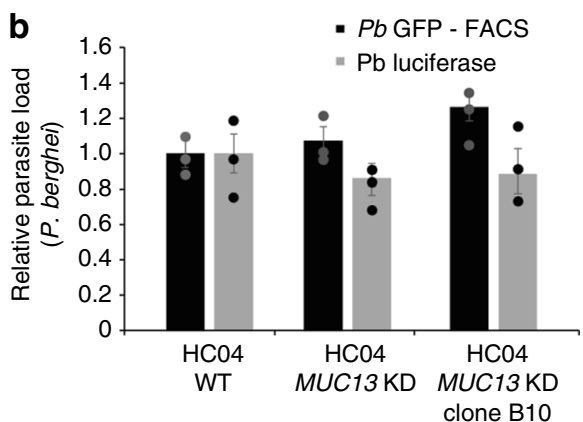

C

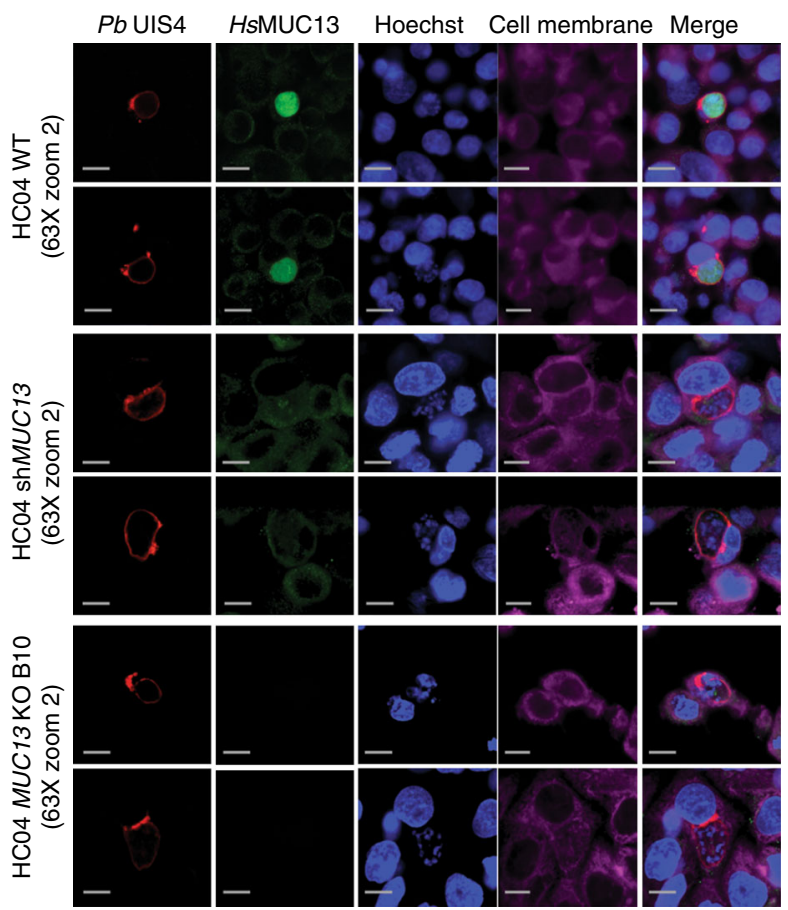

Fig. 5 Confirmation of antibody specificity in regards to HsMUC13's localization within P. berghei during liver-stage infection. a Confirmation of both MUC13 knockdown, in cells expressing a pool of four anti-mucin13 shRNAs which have been integrated into the hepatocyte genome, and MUC13 knockout, via CRISPR/Cas9, during infection via western blot. Values indicated are relative protein expression as determined by densitometry. b Effect of MUC13 knockdown and knockout in the indicated cells infected with P. berghei expressing either luciferase (measured via total luminescence) or GFP (measured by FACS). Data is presented as mean \pm s.e.m with $n=3$ with individual biological replicates overlaid. c Confocal microscopy images of HC04 liver cells, either unmodified (WT), expressing an shRNA pool for MUC13 (KD) or with MUC13 knocked out (KO clone B10), infected with P. berghei 48 hpi. Cells were labeled with a rabbit polyclonal antibody (dilution 1:500,1 mg/ml stock) against the intracellular domain of HsMUC13 (MUC13 antibody \#1 AbCam \#Ab65109). P. berghei was detected using a UIS4 antibody (1:500 dilution, $1 \mathrm{mg} / \mathrm{ml}$ stock LS-204260, LifeSpan Biosciences). Primary antibodies were detected with a goat anti-rabbit (Alexa Fluor 488, green) and a goat anti-mouse (Alexa Fluor 647, red). Cell membranes and nuclei were stained with Hoechst 33342 (blue) and CellMask deep red (magenta), respectively. Merged images between HsMUC13, P. spp HSP70, and Hoechst shown. Scale bars $10 \mu \mathrm{m} ; 63 \times$ oil objective

transcripts listed in this manuscript, 662 transcripts had clear matches in our dataset and of these 256 transcripts were differentially regulated in our dataset $(p$ value $<0.001)$. Of the 24,550 transcripts analyzed in this study, 5934 transcripts have adjusted $p$ value of less than 0.001 . The probability of this overlap (256 of 665 transcripts) occurring by chance is $3.21 \mathrm{e}-17$. Perhaps not unexpectedly, in $\sim$ half the cases (131), the changes were in different directions. It is important to consider, that the authors were mostly looking at the expression profiles of uninfected cells, after sporozoite addition, because uninfected cells will outnumber infected ones in a bulk culture by 100:1. The group showed, for example, that thymidine kinase (TK1) was strongly induced in their sporozoite-treated bulk culture at 2 hpi relative to mocktreated, salivary gland control ( $p$ value $<1 \times 10^{-7}, 3 \times$ increase in sporozoite-treated bulk cultures), but we show this induction was likely occurring in the noninfected flow-sorted cells, vs. the infected flow-sorted cells at $48 \mathrm{hpi}$ (adjusted $p$ value $=1.06 \times 10^{-18}, 3.5 \times$ decrease in infected). These data highlight that the human response to parasite infection is likely to be conserved, regardless of parasite species. 
One concern with immune-localization studies is the specificity of the MUC13 antibody signal, which colocalizes with the Plasmodium parasite by IFA microscopy and could be due to crossreactivity with glycosylated parasites proteins that are only present at $24-48 \mathrm{hpi}$. This is unlikely due to multiple independent lines of experimental evidence. First, the signal from the MUC13 antibody is sensitive to both shRNA knockdown and CRISPR/Cas9 knockout, both of which have been used by others study MUC13 phenotypes ${ }^{47}$. Second, we observe no MUC13 mRNA during early hepatocyte infection (2 hpi) (Fig. 3) or asexual blood stage infection (Supplementary Figure 5), but do detect strong parasite HSP70 signal later during exoerythrocytic development. In addition, at intermediate points of infection (12 and $24 \mathrm{hpi})$, the MUC13 IFA intensity correlates with the dramatic transcriptional induction of MUC13 during late-stage hepatic infection. In addition, the increased level of MUC13 protein within the parasite, as compared to neighboring uninfected host cells (Figs. 2, S4, S6), is consistent with the 50-fold (for Huh7.5.1) induction of MUC13 transcript levels in infected cells at $48 \mathrm{hpi}$. Third, the pattern of MUC13 expression we observe, both in host cell and parasites, is consistent with previously reported studies of MUC13 localization $^{20}$, displaying a speckled pattern with cytoplasmic and nuclear staining in cancer cells but apical in normal epithelial cells ${ }^{41}$. Fourth, we find similar staining with two independently derived MUC13 antibodies that recognize different areas of the protein. Fifth, similar MUC13 localization is observed with different parasite species. Lastly, our western blots (Supplementary Figure 3) do not indicate additional binding to products other than the slightly greater than $50 \mathrm{kDa}$ band expected for MUC13. Our data, as well as published data, thus strongly support MUC13 specificity for both of our tested MUC13 antibodies.

How MUC13 is trafficked to the parasite cytoplasm and nucleus is an open question. Other membrane-based mucins are packaged into secretory vesicles that are targeted to the site of infection and it is possible that similar localization methods are being used here. It is possible that in other systems MUC13 is exported to the site of infection but is not observed in micrographs because this space is extracellular and protein can be washed away. Cytoplasmic and nuclear localization of MUC13 is observed in cancer cells however, where MUC13 is overexpressed $^{37}$, so potentially a similar process of MUC13 internalization is at play here. However, in our case the protein is contained inside the parasitophorous vacuole of the parasite. Mutagenesis of the different domains of the protein will be necessary to determine which protein domains (EGF domains, SEA domain, and PKC phosphorylation domain) play a role in sorting MUC13 into the parasite cytoplasm.

Under normal conditions, MUC13, like most transmembrane mucins, functions to protect cells from infection and damage by forming a barrier along the mucosal surface of those cells ${ }^{41}$. However, data from our studies as well as those of others suggest that the cellular function of MUC13 is complex, particularly when expression is induced. However, since most of the transcriptional changes in host cells, such as the upregulation of cellular energy homeostasis and glucose metabolic processes and the downregulation of translational regulation and amino acid metabolism, would seem to favor parasitic development, we hypothesize that MUC13 upregulation is to the parasite's benefit as well. However, the knockdown or removal of MUC13 appears to not affect parasite development (Fig. 5b). Therefore, we hypothesize a potential role for which MUC13 in immune cell evasion. Given the localization of MUC13 throughout the developing merosome, it appears that MUC13 may be colocalized to the membranes of the developing merozoites. This could represent a parasite strategy to evade the host-cell immune system by covering the parasite membrane in host-cell glycoproteins.
This strategy has been demonstrated in several other human parasites, most notably Trypanosoma cruzi, where the parasite surrounds itself with mucin-like glycoproteins to resemble to confuse the host immune system ${ }^{48,49}$. Additionally, numerous helminths, including Schistosoma mansoni, express mucin-like molecules on their exterior to evade immune detection and aid invasion (reviewed in ref. ${ }^{50}$ ). Covering the surface of emerging merozoites with a host glycoprotein would allow those merozoites to more freely evade the immune system immediately after hepatocyte rupture and allow for more efficient erythrocyte invasion.

A final question is whether the MUC13 could be a clinically relevant biomarker of parasite infection. MUC13 protein expression has been implicated as a marker of several diseases, including numerous cancers, such as gastric, ovarian and colon cancer, inflammation and Helicobacter infection ${ }^{21,36,38}$, suggesting that MUC13 may be a general signal of cellular infection. However, these studies do not necessarily preclude the use of MUC13 as a biomarker of Plasmodium infection for two reasons: first, the fold-induction of MUC13 in other infectious diseases, outside of cancer, is much smaller than observed in this study (1.5-3 fold within the specific tissue assayed $)^{21,36}$, and second, the clinical overlap between metastatic cancer patients, where MUC13 is often highly overexpressed, and malaria patients might be small. Work on MUC13 in cancer has shown that it can be detected in peripheral blood ${ }^{38}$, suggesting that this host-factor could also potentially be used in malaria detection using peripheral blood as well. In addition, because of its role in parasite exoerythrocytic maturation, and since MUC13 is not essential gene, at least in mice ${ }^{36}$, MUC13 might be a target for antimalarial prophylaxis as well.

In summary, the identification of MUC13 as a new host factor involved in parasite development will greatly enhance our understanding of parasite liver-stage development, serve as a valuable experimental window into exoerythrocytic development and potentially could serve as a clinically relevant biomarker as well.

\section{Methods}

Parasites. P. berghei-ANKA-GFP-Luc-SMCON (Pb-Luc) and P. berghei-GFP ( PbGFP) sporozoites were obtained by dissection of infected Anopheles stephensi mosquito salivary glands. Dissected salivary glands were homogenized in a glass tissue grinder and filtered twice through nylon cell strainers $(20 \mu \mathrm{m}$ pore size, Millipore SCNY00020) and counted using a Neubauer hemocytometer. The sporozoites were kept on ice until needed. Both Pb-Luc- and Pb-GFP-infected $A$. stephensi mosquitoes were obtained from the Insectary Core Facility at New York University.

Cell culture. HC04, Huh7.5.1, and HepG2 cells were obtained from, and initially validated by, ATCC. Subsequently, cells were validated in study by microscopy as all three cell lines, HepG2, Huh7.5.1, and HC04, possess quite distinct morphology. All cell lines were cultured at $37^{\circ} \mathrm{C}$ and $5 \% \mathrm{CO}_{2}$ in DMEM (Invitrogen, Carlsbad, USA) supplemented with $10 \%$ fetal bovine serum (FBS), $0.29 \mathrm{mg} / \mathrm{mL}$ glutamine, 100 units of penicillin, and $100 \mu \mathrm{g} / \mathrm{mL}$ streptomycin. During and after the infection, cell medium was supplemented with antibiotics $50 \mu \mathrm{g} / \mathrm{mL}$ gentamycin (Gemini Bio-Products), $50 \mu \mathrm{g} / \mathrm{mL}$ neomycin trisulfate salt hydrate (SIGMA), 100 units of penicillin (SIGMA) and $100 \mu \mathrm{g} / \mathrm{mL}$ streptomycin (SIGMA), as well as the antimycotics $50 \mu \mathrm{g} / \mathrm{mL}$ 5-fluorocytosine (Cayman) and $100 \mu \mathrm{g} / \mathrm{mL}$ posaconazole (Cayman) were added to the media. Cell lines were tested for mycoplasma contamination prior to being frozen down and new cell aliquots were used for each replicate

Infection of hepatocytes for RNASeq and western blot. Twenty-four hour prior to infection, hepatocytes were either seeded in 24-well (RNA extraction-120,000 per well) or 96-well plates (luciferase growth assays-30,000 per well). Hepatocytes were infected in vitro with $P$. berghei sporozoites freshly dissected from infected Anopheles stephensi mosquitoes at a ratio of 0.3 sporozoites per seeded cell. Plates were centrifuged at $330 \times g$ for $4 \mathrm{~min}$ to bring sporozoites closer to cells, and plates were then incubated at $37^{\circ} \mathrm{C} 5 \% \mathrm{CO}_{2}$ for $2 \mathrm{~h}$ to promote sporozoite invasion ${ }^{51}$. After $2 \mathrm{~h}$, the cells were washed, fresh media containing $12 \mu \mathrm{M}$ 5-fluorocytosine (Cayman), $50 \mu \mathrm{g} / \mathrm{ml}$ gentamicin sulfate (Gemini Bio-Products), and $100 \mu \mathrm{g} / \mathrm{ml}$ 
neomycin trisulfate salt hydrate (SIGMA) was added and the cells were returned to the incubator.

Isolation of RNA from infected and uninfected hepatocytes. Cells were dissociated from plates at time zero (uninfected hepatocytes and sporozoites before infection), 24 hpi (when the sporozoites have transformed into trophozoites), and $48 \mathrm{hpi}$ (when the trophozoites have transformed into liver-stage schizonts), by the addition of $500 \mu \mathrm{L}$ TrypLE (Thermo Fisher Scientific), washed and resuspended in FACS buffer (phosphate-buffered saline (PBS) supplemented with $1 \mathrm{mM}$ ethylenediaminetetraacetic acid, $25 \mathrm{mM}$ HEPES, and $0.5 \%$ FBS), and passed through a $40 \mu \mathrm{m}$ cell strainer (Falcon). Uninfected cells were isolated from infected cells by FACS sorting with a BD Influx cell sorter, with gating based upon GFP mean fluorescence intensity (MFI). Overall gating strategy is as indicated in Supplementary Figure 8, with total cells identified via FSC vs. SSC (P1 gate), single cells identified vs FSC-height vs. FSC-width and SSC-height vs. SSC-width (gates P2 and P3), then GFP positive and negative cell collected as indicated (gates P4 and P5, respectively). Cells were sorted directly into $600 \mu \mathrm{l}$ Qiazol reagent (Qiagen) and total RNA was isolated using a Qiagen miRNEasy kit (Qiagen).

Dual RNA-sequencing. Total RNA was assessed for quantity and quality using an Agilent Tapestation. RNA libraries were generated using Illumina's TruSeq Stranded Total RNA Sample Prep Kit using at least $100 \mathrm{ng}$ of RNA. RNA libraries were multiplexed and sequenced with 100 base pair (bp) paired single end reads (SR100) to a depth of approximately 25 million reads per sample on an Illumina HiSeq2500.

Dual RNA-sequencing analysis and code availability. FastQC ${ }^{52}$ was used to perform quality control. Raw reads were first aligned to the human genome (GRCh38, release 25) using STAR2.5.2 $\mathrm{b}^{53}$ and sorted using samtools $1.2^{54}$. Gene expression was quantified using HTSeq $^{55}$. Unmapped reads were extracted using Picard tools1.141 (http://broadinstitute.github.io/picard) and aligned to P. berghei (release 32 in PlasmoDb). Parasite gene expression was obtained using the same workflow as the human transcripts. Differential expression analysis was performed using DESeq $2^{56}$. A multifactorial design was used for the paired differential expression analysis for both infected and noninfected samples at $48 \mathrm{hpi}$.

Gene validation (qPCR and western). For real-time qPCR, RNA was extracted as indicated above, then converted to cDNA using superscript II (Invitrogen) and random hexamers, according to the suggested protocol. qPCR was performed on a Bio-Rad CFX96, using the Perfecta SYBR green master mix (Quanta) and primers indicated in Supplementary Table 1. For western blots, Huh7.5.1 and HC04 cells were plated at a concentration of 120,000 cells per well in a 24 -well plate. After infection and sorting as described above, 1000 infected or 100,000 uninfected cells were washed twice with cold $1 \times$ PBS. Cells were then lysed within the plate via the addition of $200 \mu \mathrm{l}$ RIPA buffer (Teknova) plus 1:100 protease inhibitor (HaltThermo Fisher Scientific). Equal numbers of cells, due to the low numbers of infected cells, were loaded for protein analyses, and proteins were loaded onto BioRad anyKD gels. Proteins were transferred to membranes and were probed with primary anti-HsMUC13 (1:1000 Dilution from a $1 \mathrm{mg} / \mathrm{ml}$ stock, Rabbit polyclonal -Lifespan Biosciences \#LS-C345092) and $\alpha$-Vinculin (1:1000 dilution from a $1 \mathrm{mg} / \mathrm{ml}$ stock, Rabbit monoclonal-Abcam \#ab129002) antibodies at 1:1000 dilution overnight at $4{ }^{\circ} \mathrm{C}$, probed with goat anti-rabbit HRP secondary (1:5000 dilution from a $1 \mathrm{mg} / \mathrm{ml}$ stock, Life Technologies \#G21234) and detected using SuperSignal West Pico and Femto (4:1 ratio Pico:Femto) Chemiluminescent Substrate (Thermo Fisher Scientific). Densitometry was calculated using Image (http://rsbweb.nih.gov/ij/) after image inversion and is shown relative to loading control.

Plasmodium exoerythrocytic form culture for localization. For $P$. berghei imaging, 96-well glass bottom plates (MatTek Corporation) were coated with poly-Llysine $0.01 \%(\mathrm{v} / \mathrm{v})$ (SIGMA) and subsequently seeded with Huh7.5.1 or HC04 cells (110,000 cells per well) $24 \mathrm{~h}$ before infection. $P$. berghei sporozoites were freshly isolated from infected An. stephensi mosquitoes as above and resuspended in DMEM media, but additionally supplemented with $0.5 \mu \mathrm{M}$ posaconazole (Cayman), $12 \mu \mathrm{M}$ 5-fluorocytosine (Cayman), $50 \mu \mathrm{g} / \mathrm{ml}$ gentamicin sulfate (Gemini BioProducts), and $100 \mu \mathrm{g} / \mathrm{ml}$ neomycin trisulfate salt hydrate (SIGMA). Pre-seeded well plates were infected with $P$. berghei sporozoites using a 1:3 infection ratio (sporozoite to cell) and incubated for $2 \mathrm{~h}$ at $37^{\circ} \mathrm{C}$ in $5 \% \mathrm{CO}_{2}$. After $2 \mathrm{~h}$, media was replaced and plates were incubated for $48 \mathrm{~h}$.

For $P$. vivax imaging, $P$. vivax sporozoites were freshly isolated from infected An. darlingi mosquitoes from a laboratory-established colony in the Peruvian Amazon region ${ }^{43}$. P. vivax EEF culture was performed as follows, based upon previous studies ${ }^{57}$. Beginning 24 h prior to mosquito dissection and infection, 8 well Nunc Lab-Tek chamber slides (Thermo Scientific) were coated with poly-Llysine $0.01 \%$ (v/v) (SIGMA) and seeded with HC04 cells (45,000 cells per well). Accudenz purified $P$. vivax sporozoites were diluted in antibiotics and antifungals supplemented DMEM as above. Slides were infected using a 1:2 infection ratio (sporozoite to $\mathrm{HC} 04$ cell) and incubated for $4 \mathrm{~h}$ at $37^{\circ} \mathrm{C}$ under $5 \% \mathrm{CO}_{2}$ atmosphere. After this initial incubation period, infection media was replaced, and slides were incubated for 7 days, with cell culture media replaced every $48 \mathrm{~h}$

Plasmodium EEF and MUC13 localization and imaging. For MUC13 antibody \# 1 (Ab65109, AbCam), we used HC04-MUC13 wild type, HC04-MUC13 knockdown and HC04-MUC13 knockout cells and either the P. berghei Luc or P. berghei GFP rodent malaria parasites. We seeded the cells in 8-well chamber slides and infected them with a ratio of 2:1 (cells/sporozoites). At 48 hpi, the cells were fixed with $4 \%$ paraformaldehyde-PBS (Affymetrix) for $20 \mathrm{~min}$ at room temperature, permeabilized with $0.1 \%$ tritonX-100 (SIGMA) for $10 \mathrm{~min}$ at room temperature, blocked with $1 \%$ bovine serum albumin and stained overnight at $4{ }^{\circ} \mathrm{C}$ using two antibodies. The first antibody was a UIS4 (Plasmodium berghei UIS4) goat polyclonal antibody (dilution 1:500 from a $2 \mathrm{mg} / \mathrm{ml}$ stock, LS-C204260, LifeSpan BioSciences, Inc.), the second antibody was HsMUC13 (human Mucin 13, c-terminus region) rabbit polyclonal antibody (dilution 1:500 from a $1 \mathrm{mg} / \mathrm{ml}$ stock, Ab65109, AbCam). Then, the following secondary antibodies and dilutions were used (1) Rhodamine Red $^{\text {m' }}$-X (RRX AffiniPure Fab Fragment Bovine Anti-Goat IgG Fc fragment specific (Jackson ImmunoResearch Lab, Inc. \#805-297-008)) (dilution 1:1000) and (2) Alexa Fluor 488-conjugated AffiniPure Goat Anti-Rabbit IgG, Fc Fragment (Jackson ImmunoResearch Lab, Inc. \#111-545-046) (dilution 1:1000) for $2 \mathrm{~h}$ at room temperature.

Hepatocyte plasma membrane was detected using CellMask deep red (Thermo Fisher Scientific) at $1 \times$. After IFA staining, chambers were removed from $P$. berghei-infected Lab-Tek systems, slides were mounted with Vectashield with DAPI (Vector Labs) and \#1.5 glass coverslips were affixed using nail polish. Images were acquired using a Zeiss LSM880 with Airyscan Confocal Microscope $(63 \times$ oil immersion lens); diode laser power was set to $5 \%$ for $405,488,561$, and $640 \mathrm{~nm}$. Exposure values of 474 and $611 \mathrm{~ms}$ were used on the green and red channels, respectively. The images were captured and processed using the confocal ZEN software (Blue and Black edition, Zeiss).

For MUC13 antibody \#2 and \#3 (LifeSpan BioSciences \#LS-C345092 (discontinued) and LifeSpan BioSciences \#LS-A8191, respectively), we used HC04MUC13 wild type, HC04-MUC13 knockdown and HC04-MUC13 knockout cells and either the $P$. berghei Luc or $P$. berghei GFP rodent malaria parasites ${ }^{58}$. Slide chambers and 96-well plates, generated as described above, were fixed with $4 \%$ paraformaldehyde-PBS (Affymetrix) for $20 \mathrm{~min}$, permeabilized with $0.1 \%$ Triton $\mathrm{X}-100$ (SIGMA) for $5 \mathrm{~min}$ and stained overnight at $4{ }^{\circ} \mathrm{C}$ using one of three antibodies. The first antibody was a HSP70 (Plasmodium heat shock protein 70) mouse polyclonal antibody (dilution 1:500,1 mg/ml stock), developed by GenScript using a codon-optimized sequence of $P$. berghei HSP70 (PBANKA 0711900.1).

The identity of this amino acid sequence between P. berghei and P. vivax is $95 \%$ and the antibody recognizes HSP70 from both Plasmodium species. The following amino acid sequence was used to generate the antibody:

MVGGSTRIPK IQTLIKEFFN GKEACRSINP DEAVAYGAAV QAAILSGDQS NAVQDLLLLD

VCSLSLGLET AGGVMTKLIE RNTTIPAKKS QIFTTYADNQ PGVLIQVYEG ERALTKDNNL

LGKFHLDGIP PAPRKVPQIE VTFDIDANGI LNVTAVEKST GKQNHITITN DKGRLSPEEI

DRMVNDAEKY KAEDEENKKR IEARNSLENY CYGVKSSLED QKIKEKLQPN EVETCMKSVT

SILEWLEKNQ LAKDEYEAK QKEAEAVCSP IMSKIYQDAG AAAGGMPGGM PGGMPGGMPG GMPGGMNFPG GMPGGMGAPA GAPAGSGPTV EEVD.

The conserved HSP70 fragment, which was codon optimized for Escherichia coli, was expressed in E. coli, double purified, and used for mice immunization according to GenScript protocols. The second antibody was a commercially obtained PbUIS4 ( $P$. berghei upregulated in infective sporozoites gene 4) goat polyclonal antibody, used at a 1:200 dilution from $1 \mathrm{mg} / \mathrm{ml}$ (Biorbyt \#orb11636). The third antibody was HsMUC13.Two additional MUC13 antibodies, one specific for c-terminus region (rabbit polyclonal antibody, used at a 1:500 dilution from a 1 $\mathrm{mg} / \mathrm{ml}$ stock-MUC13 Antibody \#2-LifeSpan BioSciences \#LS-C345092 (discontinued)) and a second antibody specific for the extracellular domain of HsMUC13 (used at a 1:500 dilution from a $1 \mathrm{mg} / \mathrm{ml}$ stock, MUC13 Antibody \#3LifeSpan BioSciences \#LS-A8191) were also used for confirmation, using the same experimental conditions, in Supplementary Figure 6. All primary antibodies concentrations were determined by performing 15 serial dilutions $(1 \mathrm{mg} / \mathrm{ml}$ stock concentration) ranging between $1: 10$ and $1: 1 \times 10^{6}$ and a fixed concentration of secondary antibodies $(1.5 \mathrm{mg} / \mathrm{ml}$ stock concentration, dilution 1:500).

Once the primary antibody concentrations were optimized, 4 different secondary dilutions, starting from a $1.5 \mathrm{mg} / \mathrm{ml}$ stock concentration were tested: $1: 500,1: 600,1: 1000$, and 1:1500. The following secondary antibodies and dilutions were ultimately used: (1) Alexa Fluor 488-conjugated AffiniPure Goat Anti-Rabbit IgG, Fc Fragment (Jackson ImmunoResearch Lab Inc. \#111-545-008) (dilution 1:600) and (2) Alexa Fluor 647-conjugated AffiniPure Goat Anti-Mouse IgG, Fc Fragment (Jackson ImmunoResearch Lab Inc. \#111-605-008) (dilution 1:500) and (3) Alexa Fluor 647-conjugated AffiniPure Fab Fragment Bovine Anti-Goat IgG, Fc fragment specific (Jackson ImmunoResearch Lab Inc. \# 805-607-008) (dilution 1:500). Nuclei and hepatocyte plasma membrane were detected using Hoechst 33342 (Thermo Fisher Scientific) at $500 \mu \mathrm{M}$ and CellMask deep red (Thermo Fisher Scientific) at $1 \times$. After immunofluorescence staining, chambers were removed from $P$. vivax infected Lab-Tek systems, slides were mounted with 
Vectashield (Vector Labs) and \#1.5 glass coverslips were affixed using nail polish. $P$. berghei-infected well plates were resuspended in PBS and covered with aluminum foiled seals. Images were acquired using a PerkinElmer UltraView Vox Spinning Disk Confocal (100× or $60 \times$ oil objective); UltraVIEW laser power was set to $50 \%$ for $405 \mathrm{~nm}, 488 \mathrm{~nm}, 561 \mathrm{~nm}$, and $640 \mathrm{~nm}$. Exposure values of 474 and $611 \mathrm{~ms}$ were used on the green and red channel, respectively. Some 96-well plates were also scanned using the Operetta high content imaging system (PerkinElmer) (Supplementary Table 2).

Liver-stage $\boldsymbol{P}$. berghei drug in vitro assay. In vitro drug assays were performed in coated 96-well glass bottom plates (MatTek Corporation) seeded with HC04 cells and infected with $P$. berghei sporozoites as previously described. Infected wells were treated with 4-point serial 10 -fold dilutions $(1 \mu \mathrm{M}$ highest concentration) of atovaquone (Santa Cruz biotechnology \# sc-217675) or puromycin dihydrochloride (Santa Cruz biotechnology \# sc-108071B). Independent drug treatments were performed 2,12, and 24 hpi. Positive controls of growth with DMSO $0.1 \%(\mathrm{v} / \mathrm{v})$ and uninfected wells were also included. Plates were incubated for $48 \mathrm{~h}$, fixed with PBS-paraformaldehyde and stained as previously described. P. berghei EEF quantification was performed by indirect immunofluorescence using the Operetta High Content Screening system (PerkinElmer). Images were collected using a 40x objective and the acquisition parameters described in Supplementary Table 2. Parasites EEFs, hepatocytes, and nuclei were labeled using a P.sppHSP70 mouse polyclonal antibody (dilution 1:500, $1 \mathrm{mg} / \mathrm{ml}$ stock), $H s \mathrm{MUC13}$, c-terminus region rabbit polyclonal antibody (dilution 1:500,1 mg/ml stock) (LifeSpan BioSciences \#LS-C345092), and Hoechst 33342, respectively. Primary antibody detection was performed with goat anti-mouse (Alexa Fluor 647, red) and anti-rabbit (Alexa Fluor 488, green) secondary antibodies. Images were analyzed using the Harmony 3.5 Software (PerkinElmer). Objects likely to be parasites, hepatocytes, or nuclei were identified based on fluorescence intensity, and morphology. Cell area $\left(\mu \mathrm{m}^{2}\right)$ was calculated using the measure ellipse feature from Harmony.

Knockdown of MUC13. MUC13 was knocked down using an shRNA pool, containing three shRNA hairpins, targeting $M U C 13$ packaged in a lentiviral vector (Cat \# sc-45690-V, Santa Cruz Biotechnology). HC04 and Huh7.5.1 cells were plated at 120,000 cells per well in a 24 -well plate, then $24 \mathrm{~h}$ after plating media was replaced with complete media (as above) plus $5 \mu \mathrm{g} / \mathrm{ml}$ polybrene. Virus was added at a multiplicity of infection of 0.5 , cells were spun at $800 \times \mathrm{g}$ for $30 \mathrm{~min}$, then incubated overnight at $37^{\circ} \mathrm{C}$. The following day media was changed to complete media and cells were again incubated overnight at $37^{\circ} \mathrm{C}$. Cells were then moved to a 6-well plate and selected using complete media plus $2.5 \mu \mathrm{g} / \mathrm{ml}$ puromycin. After 7 days of selection, splitting as required, and knockdown efficiency was confirmed via western blot.

Knockout of MUC13. MUC13 was knocked out using an single plasmid CRISPRCas9 system, within the LV01 U6-gRNA:EF1a-puro-2A-CAS9-2A-tGFP, targeting MUC13 packaged in a lentiviral vector (Sigma-Aldrich). The gRNA sequence used was CATCTTGGCAAGGATTGCTGGG. HC04 cells were plated at 120,000 cells per well in a 24-well plate, then $24 \mathrm{~h}$ later media was replaced with complete media (as above) plus $5 \mu \mathrm{g} / \mathrm{ml}$ polybrene. Virus was added at a multiplicity of infection of 0.5 , cells were spun at $800 \times g$ for $30 \mathrm{~min}$, then incubated overnight at $37^{\circ} \mathrm{C}$. The following day media was changed to complete media and cells were again incubated overnight at $37^{\circ} \mathrm{C}$. The following day, cells were selected for presence of the CAS9 plasmid using complete media plus $2.5 \mu \mathrm{g} / \mathrm{ml}$ puromycin. After 14 days of selection, splitting as required, cells were trypsinized and plated at an average density of 1 cell per well in a 96-well plate in the presence of puromycin as above. Once cells had regrown after 24 days, wells were observed via microscopy to identify those wells which appeared to contain single colonies. The presence of MUC13 knockout within these apparent monoclonal HC04 cell lines was assessed first via PCR, then confirmed, after being grown up in $25 \mathrm{~cm}^{2}$ vented tissue culture flasks, via western blot using the Abcam MUC13 rabbit polyclonal antibody \#Ab65109. Three knockout clones (clones E8, B7 and B10) were then examined using confocal microscopy, of which two (B7 and B10) were found to have no MUC13 signal as reported within this study.

Ethics statement. Human subject protocols were approved by the Human Research Protection Program of the University of California San Diego (Approval number 120652) and Universidad Peruana Cayetano Heredia (R-157-13-14). Written informed consent was obtained from all study participants.

Reporting summary. Further information on experimental design is available in the Nature Research Reporting Summary linked to this article.

\section{Data availability}

All RNA sequencing files were deposited in the short read sequence archive (http:// www.ncbi.nlm.nih.gov/sra) under BioProject ID PRJNA390648. The source data for Fig. 1a is included here as Supplementary Data 3. The external source data used to compare between our dataset and other studies in Supplementary Data 2 are published in Tarun et al. 2008 (PMID: 18172196, PMCID: PMC2224207). All statistics for these sequencing runs are available in Supplementary Data 1. The authors declare that all other data supporting the findings of this study are available within the article and its Supplementary Information files, or are available from the authors upon request.

Received: 11 January 2018 Accepted: 24 December 2018 Published online: 30 January 2019

\section{References}

1. W.H.O. World Malaria Report: 2016 (2017).

2. Sturm, A. et al. Manipulation of host hepatocytes by the malaria parasite for delivery into liver sinusoids. Science 313, 1287-1290 (2006).

3. Olotu, A. et al. Seven-year efficacy of RTS, S/AS01 malaria vaccine among young African children. New Engl. J. Med. 374, 2519-2529 (2016).

4. Burrows, J. N., van Huijsduijnen, R. H., Mohrle, J. J., Oeuvray, C. \& Wells, T. $\mathrm{N}$. Designing the next generation of medicines for malaria control and eradication. Malar. J. 12, 187 (2013).

5. Melo, M. B. et al. Transcriptional analysis of murine macrophages infected with different Toxoplasma strains identifies novel regulation of host signaling pathways. PLoS Pathog. 9, e1003779 (2013).

6. Saeij, J. P. et al. Toxoplasma co-opts host gene expression by injection of a polymorphic kinase homologue. Nature 445, 324-327 (2007).

7. Melo, M. B., Jensen, K. D. \& Saeij, J. P. Toxoplasma gondii effectors are master regulators of the inflammatory response. Trends Parasitol. 27, 487-495 (2011).

8. Taylor, S. et al. A secreted serine-threonine kinase determines virulence in the eukaryotic pathogen Toxoplasma gondii. Science 314, 1776-1780 (2006).

9. Saeij, J. P. et al. Polymorphic secreted kinases are key virulence factors in toxoplasmosis. Science 314, 1780-1783 (2006).

10. Rosowski, E. E. et al. Strain-specific activation of the NF-kappaB pathway by GRA15, a novel Toxoplasma gondii dense granule protein. J. Exp. Med. 208, 195-212 (2011)

11. Jensen, K. D. et al. Toxoplasma gondii rhoptry 16 kinase promotes host resistance to oral infection and intestinal inflammation only in the context of the dense granule protein GRA15. Infect. Immun. 81, 2156-2167 (2013).

12. Ingmundson, A., Alano, P., Matuschewski, K. \& Silvestrini, F. Feeling at home from arrival to departure: protein export and host cell remodelling during Plasmodium liver stage and gametocyte maturation. Cell. Microbiol. 16, 324-333 (2014).

13. Kaushansky, A. et al. Malaria parasites target the hepatocyte receptor EphA2 for successful host infection. Science 350, 1089-1092 (2015).

14. Yalaoui, S. et al. Hepatocyte permissiveness to Plasmodium infection is conveyed by a short and structurally conserved region of the CD81 large extracellular domain. PLoS Pathog. 4, e1000010 (2008).

15. Orito, Y. et al. Liver-specific protein 2: a Plasmodium protein exported to the hepatocyte cytoplasm and required for merozoite formation. Mol. Microbiol. 87, 66-79 (2013).

16. Ingmundson, A., Nahar, C., Brinkmann, V., Lehmann, M. J. \& Matuschewski, K. The exported Plasmodium berghei protein IBIS1 delineates membranous structures in infected red blood cells. Mol. Microbiol. 83, 1229-1243 (2012).

17. Singh, A. P. et al. Plasmodium circumsporozoite protein promotes the development of the liver stages of the parasite. Cell 131, 492-504 (2007).

18. Cloney, R. Microbial genetics: dual RNA-seq for host-pathogen transcriptomics. Nat. Rev. Genet. 17, 126-127 (2016).

19. Matuschewski, K. et al. Infectivity-associated changes in the transcriptional repertoire of the malaria parasite sporozoite stage. J. Biol. Chem. 277, 41948-41953 (2002).

20. Sheng, Y. H. et al. MUC13 protects colorectal cancer cells from death by activating the NF-kappaB pathway and is a potential therapeutic target. Oncogene 36, 700-713 (2017).

21. Liu, C. et al. Gastric de novo Muc13 expression and spasmolytic polypeptideexpressing metaplasia during Helicobacter heilmannii infection. Infect. Immun. 82, 3227-3239 (2014).

22. Gazzinelli, R. T., Kalantari, P., Fitzgerald, K. A. \& Golenbock, D. T. Innate sensing of malaria parasites. Nat. Rev. Immunol. 14, 744-757 (2014).

23. Westermann, A. J., Barquist, L. \& Vogel, J. Resolving host-pathogen interactions by dual RNA-seq. PLoS Pathog. 13, el006033 (2017).

24. Dillon, L. A. et al. Simultaneous transcriptional profiling of Leishmania major and its murine macrophage host cell reveals insights into host-pathogen interactions. BMC Genom. 16, 1108 (2015).

25. Pittman, K. J., Aliota, M. T. \& Knoll, L. J. Dual transcriptional profiling of mice and Toxoplasma gondii during acute and chronic infection. BMC Genom. 15, 806 (2014).

26. Swann, J. et al. High-throughput luciferase-based assay for the discovery of therapeutics that prevent malaria. ACS Infect. Dis. 2, 281-293 (2016). 
27. Franke-Fayard, B. et al. A Plasmodium berghei reference line that constitutively expresses GFP at a high level throughout the complete life cycle. Mol. Biochem Parasitol. 137, 23-33 (2004).

28. Sinnis, P., De La Vega, P., Coppi, A., Krzych, U. \& Mota, M. M. Quantification of Sporozoite invasion, migration, and development by microscopy and flow cytometry. Methods Mol. Biol. 923, 385-400 (2013).

29. Vaughan, A. M. et al. Complete Plasmodium falciparum liver-stage development in liver-chimeric mice. J. Clin. Investig. 122, 3618-3628 (2012).

30. Tarun, A. S. et al. A combined transcriptome and proteome survey of malaria parasite liver stages. Proc. Natl Acad. Sci. 105, 305-310 (2008).

31. Le Roch, K. G. et al. Discovery of gene function by expression profiling of the malaria parasite life cycle. Science 301, 1503-1508 (2003).

32. Tripathi, S. et al. Meta- and orthogonal integration of influenza "OMICs" data defines a role for UBR4 in virus budding. Cell Host Microbe 18, 723-735 (2015).

33. Kramer, A., Green, J., Pollard, J. Jr. \& Tugendreich, S. Causal analysis approaches in Ingenuity Pathway Analysis. Bioinformatics 30, 523-530 (2014).

34. Hanson, K. K. et al. Torins are potent antimalarials that block replenishment of Plasmodium liver stage parasitophorous vacuole membrane proteins. Proc. Natl Acad. Sci. USA 110, E2838-E2847 (2013).

35. Cheng, L. et al. The Helicobacter heilmannii hofE and hofF genes are essential for colonization of the gastric mucosa and play a role in IL-1beta-induced gastric MUC13 expression. Helicobacter 21, 504-522 (2016).

36. Sheng, Y. H. et al. The MUC13 cell-surface mucin protects against intestinal inflammation by inhibiting epithelial cell apoptosis. Gut 60, 1661-1670 (2011).

37. Gupta, B. K. et al. Functions and regulation of MUC13 mucin in colon cancer cells. J. Gastroenterol. 49, 1378-1391 (2014).

38. Maher, D. M., Gupta, B. K., Nagata, S., Jaggi, M. \& Chauhan, S. C. Mucin 13: structure, function, and potential roles in cancer pathogenesis. Mol. Cancer Res. 9, 531-537 (2011)

39. Renia, L. et al. A malaria heat-shock-like determinant expressed on the infected hepatocyte surface is the target of antibody-dependent cell-mediated cytotoxic mechanisms by nonparenchymal liver cells. Eur. J. Immunol. 20, 1445-1449 (1990).

40. Mueller, A. K. et al. Plasmodium liver stage developmental arrest by depletion of a protein at the parasite-host interface. Proc. Natl Acad. Sci. USA 102, 3022-3027 (2005).

41. Williams, S. J. et al. Muc13, a novel human cell surface mucin expressed by epithelial and hemopoietic cells. J. Biol. Chem. 276, 18327-18336 (2001).

42. Gupta, B. K. et al. Increased expression and aberrant localization of mucin 13 in metastatic colon cancer. J. Histochem. Cytochem. 60, 822-831 (2012).

43. Moreno, M. et al. Infection of laboratory-colonized Anopheles darlingi mosquitoes by Plasmodium vivax. Am. J. Trop. Med. Hyg. 90, 612-616 (2014).

44. Albuquerque, S. S. et al. Host cell transcriptional profiling during malaria liver stage infection reveals a coordinated and sequential set of biological events. BMC Genom. 10, 270-270 (2009).

45. Posfai, D. et al. Plasmodium parasite exploits host aquaporin-3 during liver stage malaria infection. PLOS Pathog. 14, e1007057 (2018).

46. Chattopadhyay, R. et al. Early transcriptional responses of HepG2-A16 liver cells to infection by Plasmodium falciparum sporozoites. J. Biol. Chem. 286, 26396-26405 (2011).

47. Chauhan, S. C. et al. MUC13 mucin augments pancreatic tumorigenesis. Mol. Cancer Ther. 11, 24-33 (2012).

48. Flavia Nardy, A., Freire-de-Lima, Geraldo, C. \& Morrot, A. Immune evasion strategies of Trypanosoma cruzi. J. Immunol. Res. 2015, 7 (2015).

49. Nardy, A. F. F. R., Freire-de-Lima, L., Freire-de-Lima, C. G. \& Morrot, A. The sweet side of immune evasion: role of glycans in the mechanisms of cancer progression. Front. Oncol. 6, 54 (2016).

50. Theodoropoulos, G., Hicks, S. J., Corfield, A. P., Miller, B. G. \& Carrington, S. D. The role of mucins in host-parasite interactions: Part II-helminth parasites. Trends Parasitol. 17, 130-135 (2001).

51. Prudencio, M., Rodriguez, A. \& Mota, M. M. The silent path to thousands of merozoites: the Plasmodium liver stage. Nat. Rev. Microbiol. 4, 849-856 (2006).

52. Andrews, S. FastQC: a quality control tool for high throughput sequence data, https://www.bioinformatics.babraham.ac.uk/projects/fastqc (2010).
53. Dobin, A. et al. STAR: ultrafast universal RNA-seq aligner. Bioinformatics 29, 15-21 (2013).

54. Li, H. et al. The sequence alignment/map format and SAMtools. Bioinformatics 25, 2078-2079 (2009).

55. Anders, S., Pyl, P. T. \& Huber, W. HTSeq-a Python framework to work with high-throughput sequencing data. Bioinformatics 31, 166-169 (2015).

56. Love, M. I., Huber, W. \& Anders, S. Moderated estimation of fold change and dispersion for RNA-seq data with DESeq2. Genome Biol. 15, 550 (2014).

57. Orjuela-Sanchez, P. et al. Developing Plasmodium vivax Resources for Liver Stage Study in the Peruvian Amazon Region. ACS Infect. Dis. 4, 531-540 (2018).

58. Franke-Fayard, B. et al. Simple and sensitive antimalarial drug screening in vitro and in vivo using transgenic luciferase expressing Plasmodium berghei parasites. Int. J. Parasitol. 38, 1651-1662 (2008).

\section{Acknowledgments}

We thank the members of the Winzeler and Lewis labs for advice and critical reading of the manuscript. In addition, we thank Medicines for Malaria Venture for all of their support of the insectary in Peru. We would also like to thank the UCSD Institute for Genomic Medicine Sequencing Core Facility and the UCSD Human Embryonic Stem Cell Flow Cytometry Core Facility for their technical support. G.L. is supported by an A. P. Giannini Post-Doctoral Fellowship. E.A.W. is supported by grants from the NIH (5R01AI090141 and R01AI103058). N.E.L. and S.L. received funding from the NIGMS (R35 GM119850) and the Novo Nordisk Foundation through the Center for Biosustainability at the Technical University of Denmark (NNF10CC1016517). The P. vivax work was supported by grants to J.M.V. from the NIH (D43TW007120 and U19AI089681). A.N.C. received support from a NIH T32 AI 007036 training grant.

\section{Author contributions}

G.L., P.O.S., J.C., L.W., A.C. and E.A.W. designed the experiments and wrote the manuscript. Parasite infection, flow sorting and RNA extraction were performed by G.L., P.O.S., L.W. and J.S. Transcriptome sequencing was performed by the UCSD Institute for Genomic Medicine Core Facility. P. vivax experiments were designed by J.M.V., P.O.S., J. C. and M.M. and performed by P.O.S., Z.V.G., M.M. and C.T.R. Sequence analysis was performed by S.L., A.M.A. and N.L. Immunofluorescence assays were designed and performed by P.O.S., J.C. and E.A.W. Knockdown of MUC13 and subsequent determination of infection rate was performed by G.L. and B.Y.Z. All authors read and approved the manuscript.

\section{Additional information}

Supplementary Information accompanies this paper at https://doi.org/10.1038/s41467019-08349-0.

Competing interests: The authors declare no competing interests.

Reprints and permission information is available online at http://npg.nature.com/ reprintsandpermissions/

Publisher's note: Springer Nature remains neutral with regard to jurisdictional claims in published maps and institutional affiliations.

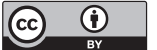

Open Access This article is licensed under a Creative Commons Attribution 4.0 International License, which permits use, sharing, adaptation, distribution and reproduction in any medium or format, as long as you give appropriate credit to the original author(s) and the source, provide a link to the Creative Commons license, and indicate if changes were made. The images or other third party material in this article are included in the article's Creative Commons license, unless indicated otherwise in a credit line to the material. If material is not included in the article's Creative Commons license and your intended use is not permitted by statutory regulation or exceeds the permitted use, you will need to obtain permission directly from the copyright holder. To view a copy of this license, visit http://creativecommons.org/ licenses/by/4.0/.

(C) The Author(s) 2019 